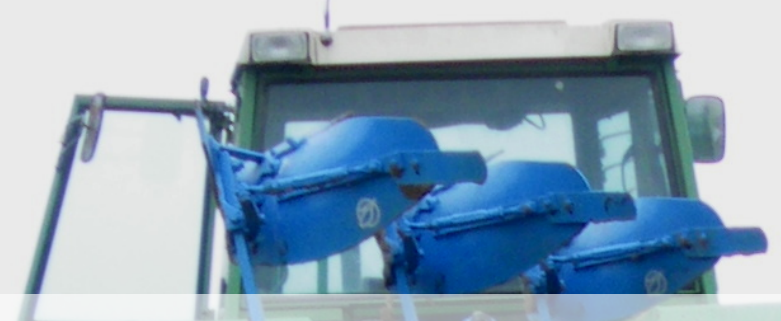

Volume 2 (2016) | Issue 1

Basel, Switzerland ISSN: 2297-6485

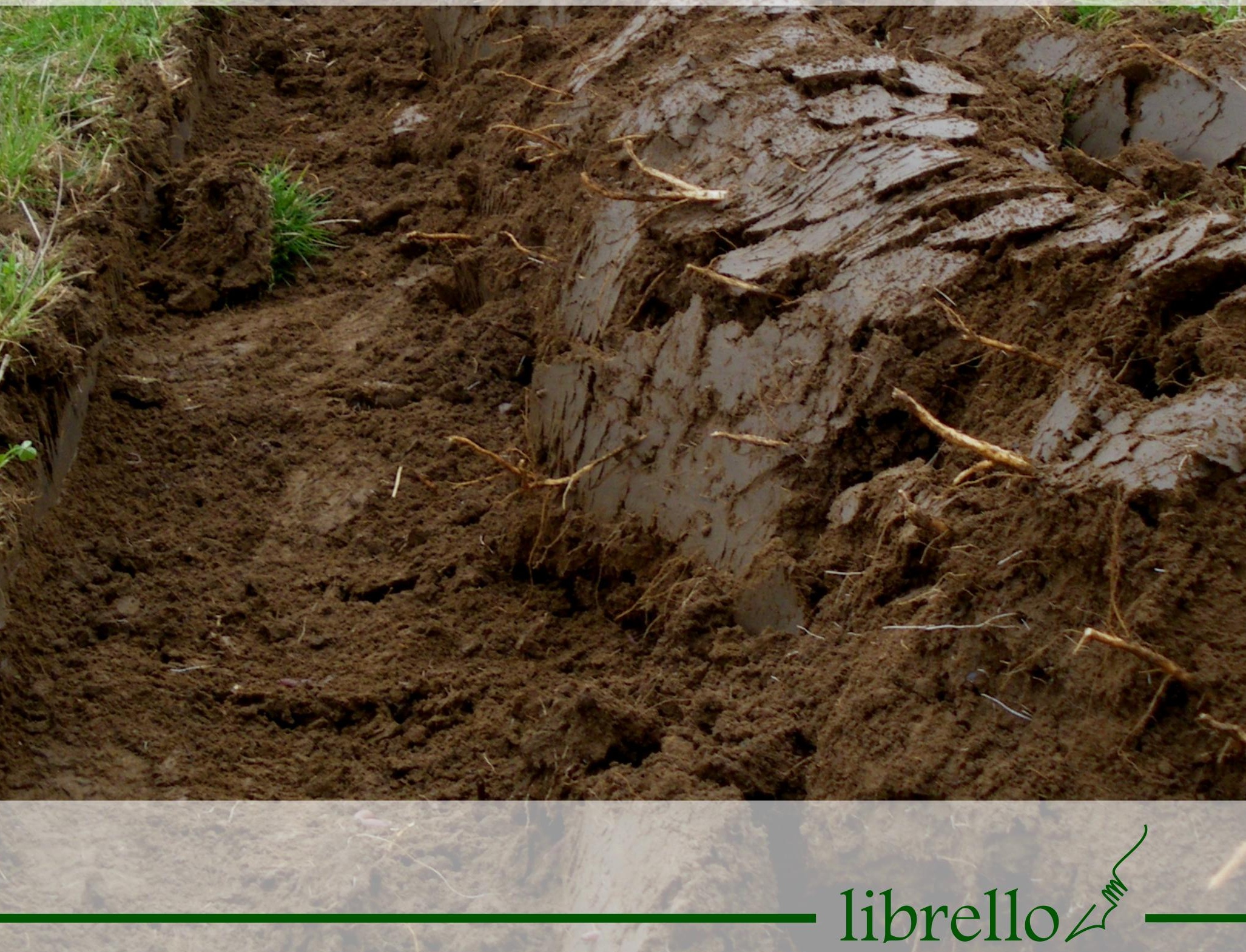


Organic Farming | 2016 | Volume 2 | Issue 1

Organic Farming is an international, open access, academic, interdisciplinary journal, published by Librello.

\section{Cover image}

Spring tillage of a two-year multispecies legume-grass for a cropping-system experiment at the organic farm Wilmersdorf in northeast Germany (author: Ralf Bloch). 


\section{About Organic Farming}

\section{Focus \& Scope}

Organic Farming (OF; ISSN 2297-6485) is a new open access academic journal that publishes articles on advances and innovations in organic agriculture and food production to provide scholars and other groups with relevant and highly topical research in the field.

Organic Farming is a new open access academic journal that publishes articles on advances and innovations in organic agriculture and food production to provide scholars and other groups with relevant and highly topical research in the field.

Organic Farming welcomes contributions in diverse areas related to organic farming and food production, such as soil and plant management, crop breeding, regulation of pests and diseases, protection of soil, water, biodiversity and other resources, livestock health and management, marketing and acceptance of organic products, food quality and processing, policies and regulations.

The articles of Organic Farming will be immediately accessible upon publication and we aim at making this journal a valuable venue for the communication among scientists, but also between researchers, producers, policy makers, traders and consumers of organic products.

Topics covered by this journal include, but are not limited to: agroforestry systems; biodiversity; biological pest and disease control; certification and regulation; compost and manure management; consumer research; crop rotations; ecosystem services; food processing; food quality and safety; green manures; nutrient cycling and run-off; organic energy production; organic farming for food security; plant breeding and genetics; poverty eradication and human development; regulation and policies; resilience and transformations; social acceptance and marketing; soil and water protection; sustainability and ethics of livestock production; sustainable agriculture; tillage and no-till organic farming systems; veterinary aspects of organic livestock production; weed ecology and management; and related topics.

Organic Farming will specially welcome original interdisciplinary and trans-disciplinary contributions.

Indexing, abstracting and archiving

AGORA-FAO, CABI, CiteSeerX, DOAJ, EBSCO, GOOGLE Scholar, J-gate, OCLC WorldCat, ResearchBible, WorldCat

\section{(2) Organic}

http://www.librelloph.com/organicfarming 


\section{Editorial Team}

Editor-in-Chief

Thomas Döring, Humboldt University of Berlin, DE

Associate Editors:

Christian Bruns, Universität Kassel, DE

Eric Gallandt, University of Maine, USA

Johannes Kahl, Universität Kassel, DE

Susanne Padel, The Organic Research Centre, UK

Mette Vaarst, Aarhus University, DK

Managing Editor

José Alberto Fernandez Monteiro, Librello, Switzerland

\section{Editorial Board}

Hossein Azadi, Ghent University, BE

Johann Bachinger, Leibniz Centre for Agricultural Landscape Research, DE

Catherine Badgley, University of Michigan, USA

Jan Bengtsson, Swedish University of Agricultural Sciences, SE

Subrahmanyeswari Bodapati, NRT College of Veterinary Science, IN

Michael Bomford, Kwantlen Polytechnic University in British Columbia, CA

Ademir Calegari, Agricultural Research Institute of Paraná, BR

Heather Darby, University of Vermont, USA

Martine Dorais, Laval University, CA

Charles Francis, University of Nebraska, USA

Bernhard Freyer, University of Natural Resources and Life Sciences, AT

Brian B. McSpadden Gardener, Ohio State University, USA

Andreas Gattinger, Research Institute of Organic Agriculture, $\mathrm{CH}$

José Gómez, Institute for Sustainable Agriculture, ES

Parwinder Grewal, Ohio State University, USA

Niels Halberg, International Centre for Research in Organic Food Systems, DK

Michael Hauser, University of Natural Resources and Life Sciences, AT

Marcel van der Heijden, Agroscope Reckenholz-Tänikon, $\mathrm{CH}$

Anna Maria Häring, Eberswalde University for Sustainable Development, DE

Erik Steen Jensen, Swedish University of Agricultural Sciences, SE

Henning Høgh Jensen, Technical University of Denmark, DK

Karen Klonsky, University of California, USA

Radics László, Corvinus University of Budapest, HU

Matt Lobley, University of Exeter, UK

Urs Niggli, Research Institute of Organic Agriculture, $\mathrm{CH}$

Myles Oelofse, University of Copenhagen, DK

Leo van Overbeek, Wageningen University and Research Centre, NL

David Pearson, University of Canberra, AU

Angelika Ploeger, Universität Kassel, DE

Michael Raviv, Newe Ya'ar Research Center, ARO, Ramat Yishay, IL

Alexandra Stone, Oregon State University, USA

Michelle Wander, University of Illinois at Urbana-Champaign, USA

Gladis Zinati, Rodale Institute, USA

Werner J. Zollitsch, University of Natural Resources and Life Sciences, AT 


\section{Table of Contents}

Research Article ....................................................

Management Options for Organic Winter Wheat Production under Climate Change

Ralf Bloch ${ }^{1,2, *}$, Jürgen $\mathrm{He}^{3}$ and Johann Bachinger ${ }^{1}$

${ }^{1}$ Leibniz Centre for Agricultural Landscape Research (ZALF), Institute of Land Use Systems, Müncheberg, Germany

${ }^{2}$ Eberswalde University for Sustainable Development, University of Applied Sciences, Eberswalde, Germany

${ }^{3}$ University of Kassel, Witzenhausen, Department of Organic Farming and Cropping Systems, Witzenhausen, Germany

${ }^{*}$ Corresponding author

Research Article . ................................................... 17

An Experimental Test of a Biodynamic Method of Weed Suppression: The Biodynamic Seed Peppers

Bruce Kenneth Kirchoff

Department of Biology, University of North Carolina at Greensboro Greensboro, NC, USA

Book Review ......................................................21

A Review of 'Organic Struggle: The Movement for Sustainable Agriculture in the United States'

Charles Francis ${ }^{1,2}$

${ }^{1}$ Department of Agronomy \& Horticulture, University of Nebraska - Lincoln, Lincoln, NE, USA

${ }^{2}$ Plant Sciences Department, Norwegian University of Life Sciences, Ås, Norway

Research Article ..................................................... 23

Can the Adoption of Organic Farming Be Predicted by Biogeographic Factors? A French Case Study

Marco Pautasso ${ }^{1, \star}$, Anja Vieweger ${ }^{2}$ and A. Márcia Barbosa ${ }^{3}$

${ }^{1}$ Animal and Plant Health Unit, European Food Safety Authority (EFSA), Parma, Italy

${ }^{2}$ Organic Research Centre, Elm Farm, Hamstead Marshall, Newbury, UK

${ }^{3}$ Centro de Investigação em Biodiversidade e Recursos Genéticos (CIBIO), InBIO Research

Network in Biodiversity and Evolutionary Biology, University of Évora, Portugal

${ }^{*}$ Corresponding author 


\title{
Management Options for Organic Winter Wheat Production under Climate Change
}

\author{
Ralf Bloch ${ }^{1,2, *}$, Jürgen $\mathrm{He}^{3}$ and Johann Bachinger ${ }^{1}$ \\ ${ }^{1}$ Leibniz Centre for Agricultural Landscape Research (ZALF), Institute of Land Use Systems, Müncheberg, Germany \\ ${ }^{2}$ Eberswalde University for Sustainable Development, University of Applied Sciences, Eberswalde, Germany \\ ${ }^{3}$ University of Kassel, Witzenhausen, Department of Organic Farming and Cropping Systems, Witzenhausen, Germany
}

* Corresponding author: E-Mail: bloch@zalf.de; Tel.: +49 3343282423; Fax: +49 3343282387

Submitted: 25 September 2015 | In revised form: 16 March 2016 | Accepted: 19 March 2016 |

Published: 19 April 2016

\begin{abstract}
An effective adaptive strategy for reducing climate change risks and increasing agro-system resiliency is broadening cropping system diversity, heightening the flexibility of cultivation and tillage methods. Climate change impacts on standard cultivation practices such as mineralisation and nitrate leaching due to mild and rainy winters, as well as frequent drought or water saturation, not only limiting fieldwork days, but also restricting ploughing. This calls for alternative methods to counteract these propensities. From 2010 to 2013, a farming system experiment was conducted on a distinctly heterogeneous organic farm in Brandenburg, Germany. With the intention of devising a more varied and flexible winter wheat cultivation method, standard organic farming practices (winter wheat cultivation after two years of alfalfa-clover-grass and ploughing in mid-October) were compared to four alternative test methods, which were then evaluated for their robustness and suitability as adaptive strategies. Two of the alternative methods, early sowing and catch crop, entailed moving up the date for alfalfa-clover-grass tilling to July. Instead of a plough, a ring-cutter was used to shallowly $(8 \mathrm{~cm}) \mathrm{cut}$ through and mix the topsoil. In the early sowing test method, winter wheat was sown at the end of August, after repeated ring-cutter processing. With the catch crop method, winter wheat seeding followed a summer catch crop and October tillage. The two oat methods (oat/plough; oat/ring-cutter) entailed sowing winter wheat in September, following oat cultivation. Overall, the cultivation methods demonstrated the following robustness gradation: standard practice $=$ catch crop $\geq$ early sowing $>$ oat/plough $>$ oat/ring-cutter. When compared to standard procedures, the catch crop and early sowing test methods showed no remarkable difference in grain yields. Measured against early sowing, the catch crop test method was significantly more robust when it came to winterkill, quality loss, and weed infestation (40\% lower weed-cover). High $\mathrm{N}_{\text {min }}$-values (up to $116 \mathrm{~kg} \mathrm{~N} \mathrm{ha}^{-1}$ ) in autumn could have caused the chamomile and thistle infestation in both oat/plough and oat/ring-cutter test methods, which led to crop failure in the hollows. Compared to standard practices, the oat ring-cutter test method brought in over $50 \%$ less grain yield. This was attributed to ring-cutter processing, which reduced $\mathrm{N}$ mineralisation and caused high weed infestation. However, the ring-cutter effectively regulated alfalfa-clover-grass fields in both exceedingly wet and very dry weather; a temporal flexibility which increases the number of fieldwork days. The catch crop and early sowing test methods contributed most to boosting future agronomic diversity.
\end{abstract}

Keywords: Adaptive capacity; cropping systems; on-farm research; reduced tillage; winter wheat 


\section{Introduction}

Farmers today face the challenge of adapting their crop cultivation methods to climatic changes. In the near future, farms with a high adaptive capacity will have a distinct advantage. The adaptive capacity of a farm is established first and foremost by expanding diversity and flexibility [1]. Between 2009 and 2014, strategies to increase the adaptive capacity of organic farms were developed within the interdisciplinary project Innovation Network of Climate Change Adaptation Brandenburg Berlin (INKA BB; http://www.inka-bb.de/). Addressing practical issues regarding climate change adaptation, farmers and scientists worked together to develop modification measures, which were then tested on-site [2]. This paper illustrates a farming system experiment probing test methods for adapting winter wheat cultivation to climate change. The experiment was carried out from 2010 to 2013 in northeast Germany on the stockless organic farm Wilmersdorf.

\subsection{Problem Description}

Winter wheat provides the economic foundation for the Wilmersdorf organic farm and has, thus far, been cultivated solely according to standard procedures. These standard organic farming procedures entail sowing winter wheat on fields prepared with a two-year multispecies legumegrass (LGS), which is mulched two to four times a year and ploughed under in autumn of the second harvest year. Directly following this virgin tillage (i.e. ploughing the sward without prior shallow soil processing), winter wheat is sown in mid-October. The subsequent crop is usually winter rye. This standard cultivation practice is practiced on large, rolling fields averaging 40 hectares, interspersed with hills and hollows. Such small-scale heterogeneity with varying soil types (see Section 2.2), further complicates ploughing and seedbed preparation. Poor soil contact and uneven germination are common problems. Particularly during dry periods, a common occurrence at Wilmersdorf (Section 2.3), hilltops are low yield (problem) areas and ploughing the shallow topsoil there is limited. In contrast, the hollows are often waterlogged, particularly in spring and in years with heavy precipitation, which also severely limits ploughing. The farm manager reports an annual fluctuation in winter wheat yields, between $0.9-5.8 \mathrm{t} \mathrm{ha}^{-1}\left(\varnothing 3.5 \mathrm{t} \mathrm{ha}^{-1}\right)$. Therefore, to attain high winter wheat yields with the standard practice, the nitrogen $(\mathrm{N})$ supply from LGS residuals and the $\mathrm{N}$-up-take vital to winter wheat development must occur synchronously [3]. This process is closely linked to the water supply and the soil's microbial activity, which could be strongly influenced by climate changes projected for Brandenburg [4]. Such climatic developments include frequent mild and wet winters, increasing spring and summer droughts, recurrent extreme weather events (heavy rainfall and drought), and a rise in the average annual temperature [5]. Figure 1 depicts projected climatic changes from 2062 to 2092, illustrating their effects on the Wilmersdorf organic farm.
In view of these projected climate changes, the current standard cultivation practice reveals several weaknesses:

LGS virgin tilling in mid-October, followed by a mild and rainy winter, may lead to $\mathrm{N}$-mineralisation, nitrate leaching and erosion, as the low development of winter wheat mass is unable to take up sufficient nitrogen at this time [6]. On the other hand, increasing drought in early spring reduces microbial nitrogen release [7]. This can result in an $\mathrm{N}$-deficiency during developmental phases, when winter wheat has high nitrogen requirements (Stem elongation; Zadoks scale 30-32) [8]. Furthermore, ploughing in autumn heightens erosion susceptibility and the risk of plough sole compression [9]. As a result, root depth and infiltration can be retarded, hindering the soil's buffering capacity in extreme weather events (heavy rain and drought).

These issues are compounded by the influence dry periods and increased precipitation in late autumn and winter has on the number of suitable fieldwork days for ploughing and sowing. In the future, winter crop sowing conditions could deteriorate in direct proportion to the instability of appropriate fieldwork days [10].

Since LGS processes great quantities of water via transpiration, increasing temperatures can potentially lead to a water shortage in the soil, inhibiting subsequent crop growth [9]. Despite uncertainties in climate predictions, the above listed weaknesses illustrate how strongly climatic changes may influence growth, site and weather conditions, and so of course crop yields. The question is, which strategies and measures can minimise yield risk in future winter wheat cultivation on the Wilmersdorf organic farm?

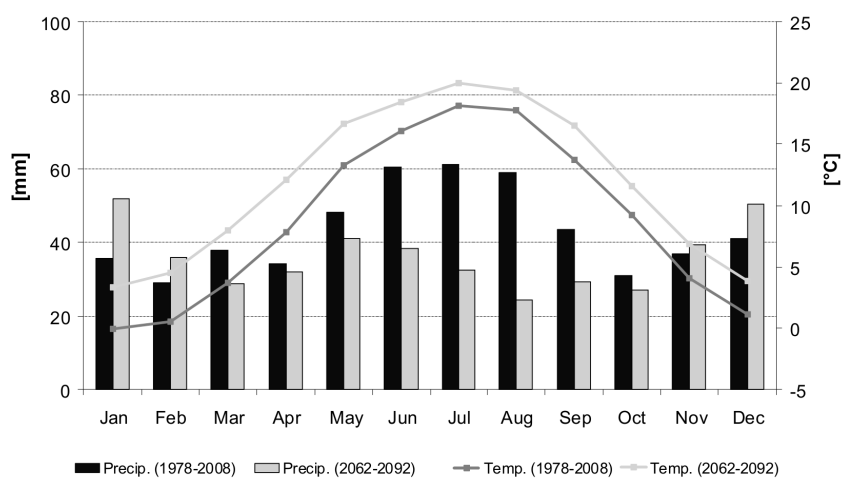

Figure 1. Monthly temperatures and precipitation observed from 1978 to 2008 and projected for 2062-2092 by the Angermünde Climate Station; Climate data modeled on the regional statistics model STARS [11].

\subsection{The Central Issue}

The extent of climate risks can be offset by increasing diversification within an agricultural ecosystem. This means not only introducing a greater diversity in crops and varieties, but also more flexibility in cultivation and tillage methods $[12,13]$. Such alternative tilling and/or seeding methods help farmers more effectively adapt to changing weather conditions, extending the number of available fieldwork days. 
To date, the Wilmersdorf organic farm has used only one method for producing winter wheat.

This work is therefore dedicated to the following questions: In addition to the standard practice, which winter wheat cultivation methods are most effective in offsetting climate change influences? Which methods contribute most to diversification on a specific farm?

\subsection{Alternative Test Methods for Winter Wheat Cultivation}

In collaboration, the Wilmersdorf farm manager and the INKA BB field trial project a) developed new cultivation techniques and, b) tested the viability of these methods as alternatives to standard procedures. When evaluating various courses of action, i.e. cultivation methods, for their adaptability to uncertain climate change conditions, robustness is an important criterion [14]. Robustness is a system's immunity to a wide range of influences [15]. In this case, the robustness of a new cultivation method is measured by its yield stability over a three-year period under varying field conditions. Hallmarks of these new cultivation methods are reduced tillage and alternative crop rotation. They should be able to improve the $\mathrm{N}$ and water supply to winter wheat. These alternative cultivation methods, (Figure 2), and the reduced tillage agricultural tool, the ring-cutter, will be described in detail.

A new agricultural tool, the ring-cutter, was used for the first time during the field tests in Brandenburg. This agricultural instrument has cutting rings running diagonally to the driving direction, allowing for an overall, non-turning, shallow tillage (see: http://www.heko-landmaschinen.de). The special ring-cutter construction is intended for soil processing when dryness or sogginess renders ploughing unsuitable. As opposed to the plough, the ring-cutter could be applied to both the dry hilltops and damp hollows on Wilmersdorf farm. The tool should also enable unploughed LGS processing, so that grain mulch seeding (early sowing and oat/ring-cutter test methods) or catch crops can be seeded directly following ring-cutter processing. If ring-cutter processing proves successful, summer crops and early sowing can stay on schedule, despite wet or dry soil. At the same time, shallow processing can reduce the water loss caused by evaporation in ploughed soil. Reduced tillage with the ring-cutter should also retard $\mathrm{N}$-mineralisation, reducing the risk of $\mathrm{N}$-leaching in winter (see test method oat/ring-cutter) [16].

\subsubsection{Early Sowing of Winter Wheat and a Summer Catch Crop}

In the cultivation methods early sowing and catch crop, the LGS processing date is moved forward to mid-July (summer processing). This early treatment of LGS can either move winter wheat seeding forward or prepare a better seedbed than virgin tillage and an autumn furrow do. Instead of the plough, the ring-cutter is used for early, mechanical LGS killing. The early sowing test method repeats ring-cutter processing two or three times between mid-July and mid-
August, creating a mulched summer fallow. This fallow protects the soil from water loss that would otherwise occur in living LGS crop transpiration [9]. The mulch also protects from evaporation, reducing water loss from the soil surface (evaporation fallow) [17], while seasonal heavy rains are buffered by the mulch (erosion protection) [18]. At the end of August, the catch crop mixture (50\% summer vetch, Vicia sativa; $28 \%$ buckwheat, Fagopyrum esculentum; 16\% flax, Linum usitatissimum; and $5 \%$ Phacelia) is sown directly into the summer fallow (mulch seeding). Not turning the earth protects soil life, keeping vertical earthworm channels (macropores) intact and improving infiltration [19-21]. Since it has a longer and stronger plant growth until its dormant season, early sown winter wheat should better absorb the nitrogen mineralised from late summer to early autumn $[22,23]$. The catch crop mixture should suppress weed growth and prevent an overly lush development of the early sown winter wheat [24]. Furthermore, in autumn the catch crop absorbs any potential nitrogen surplus, conserving it in its biomass throughout the winter. In spring, the frozen catch crop vegetal material mineralizes the soil, supplying the winter wheat with the required nitrogen. Early sown winter wheat develops more profusely in spring, creating better rooting in the subsoil. This is conducive to the nitrogen and water supply in the event of a spring drought. Also, early sowing pre-dates winter wheat primary water needs (stem elongation) to a time when the soil should still have enough winter moisture. Furthermore, the early seeding head-start in growth should assure the plants are no longer subjected to drought stress during the grain filling phase $[25,26]$. The unploughed early sowing test method should primarily improve crop establishment and the subsequent grain yield on dry hilltops.

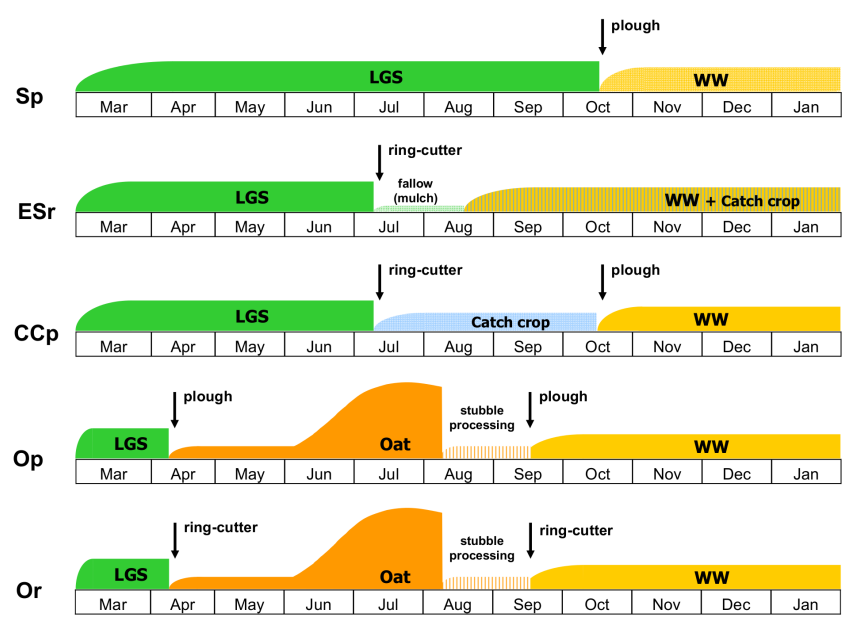

Figure 2. Winter wheat (WW) test methods with plough and ring-cutter in the cultivation field tests on the farm Wilmersdorf (2010-2013). Sp: standard practice (plough); ESr: WW early sowing (ring-cutter); CCp: winter wheat following catch crop (plough); Op: winter wheat following oat (plough) and Or: winter wheat following oat (ring-cutter); LGS: Legume-grass swards; personal compilation. 
In contrast to early sowing, the catch crop test method does not have summer fallow. Instead, after repeated processing with the ring-cutter, the same catch crop mixture as in early sowing is sown at the end of July. The aim of the catch crop is to bind nitrogen mobilised by the early LGS tilling in summer, and to convert it into organic material which will have a narrow $\mathrm{C} / \mathrm{N}$ ratio the following spring [27]. This material activates soil life, improving $\mathrm{N}$ resources for winter wheat. Coinciding with the standard autumn furrow, the catch crop is ploughed immediately before winter wheat late seeding (mid-October).

\subsubsection{Winter Wheat Following an Oat Crop}

In the oat/plough and oat/ring-cutter test methods, LGS tillage takes place in late March. The advantage of springtime tillage over autumn tillage is that the pre-winter mineralisation from LGS residuals, and so nitrate leaching, is nearly non-existent [6]. Compared to autumn tillage, spring tillage usually allows for a better balance between the $\mathrm{N}$ release from LGS residuals and $\mathrm{N}$ crop up-take [27]. Due to the generally cooler temperatures and a high C/N LGS residual ratio, tillage in spring can also go hand in hand with delayed $\mathrm{N}$ mineralisation $[6,27]$. Since the mineralisation process following spring tillage begins later and lasts longer, often only the second subsequent crop profits from the $\mathrm{N}$ provided by multi-annual LGS [6]. For this reason, the oat/plough and oat/ring-cutter test methods switch crop rotation, growing oats prior to winter wheat. By inserting oats, the N-deployment from organic plant matter (decomposed LGS residuals, straw and oat root residues) is more in sync with winter wheat $\mathrm{N}$ up-take the following spring. The advantages of oat as an LGS subsequent crop are its extensive root system and high nutrient up-take [28], while as a winter wheat preceding crop, oat's vegetative growth suppresses weeds. As a recovery crop, oat prevents the spreading of fungal pathogens, such as black root rot (Gaeumannomyces graminis) [29,30]. At the same time, the increased winter moisture resulting from climate change should be used productively for early oat cultivation, extending the cultivation period in the future. An earlier vegetation period grants a surplus of available fieldwork days for seeding spring grains [10,31].

To keep future seeding on schedule despite higher winter precipitation (see Figure 1) saturating the soil, the oat/ringcutter test method foregoes ploughing, working the oats with the ring-cutter to establish mulch-till. After harvesting the oats, the stubble is worked with the ring-cutter. The oat straw remains on the field, as its broad $\mathrm{C} / \mathrm{N}$ ratio helps prevent excessive LGS-residue $\mathrm{N}$ release in autumn and subsequent nitrate leaching in winter. In contrast to standard procedure virgin tillage, prior oat cultivation improves the winter wheat seedbed preparation and allows for a more flexible seeding schedule. In both oat test methods, winter wheat is sown at the end of September. In test method oat/ring-cutter, seeding follows reworking the soil with the ring-cutterunploughed mulch-till-while test method oat/plough en- tails renewed ploughing. All unploughed test methods are designed to improve dry hilltop yields.

\subsubsection{Hypotheses}

The robustness of each cultivation method is tested based on the following hypotheses:

(1) The ring-cutter effectively cuts and regulates LGS in both damp and dry soil conditions, where ploughing is restricted.

(2) Compared to the standard practice, winter wheat test methods ploughed in autumn, catch crop and oat/plough, produce equivalent annual grain yields, on hilltops as well as in hollows.

(3) The unploughed winter wheat test methods early sowing and oat/ring-cutter are particularly suitable for dry hilltop sites, where, when compared to hollows, the annual grain yields of these test methods come closer to those of standard practices.

\section{Materials and Methods}

\subsection{Farm Location and Characteristics}

The stockless organic farm Wilmersdorf (Bioland growers' association member) is located in the north German lowlands in the northeast of Brandenburg (Uckermark; community Angermünde, district of Wilmersdorf: $53.11431^{\circ} \mathrm{N}$; $\left.13.90660^{\circ} \mathrm{E}\right)$. The farm has over 1,108 hectares of arable land and 5 hectares of grassland. Almost half of the arable land is used to grow legumes. Forage legume cultivation accounts for the main area (surface area 29\%), followed by grain legume cultivation (19\%). The remaining half of the arable land is reserved for growing grains, winter rye $(28 \%)$ and winter wheat $(20 \%)$, spelt $(16 \%)$ and spring wheat $(15 \%)$, as well as winter and spring barley $(12 \%$ and $9 \%$, respectively). Wilmersdorf has predominately sandy loamy soil and implements the following crop rotation: Alfalfa clover-grass / winter wheat / winter rye / field peas / spelt under sown with alfalfa clover-grass.

\subsection{Soil Characteristics}

Wilmersdorf, located in the upper moraine region, is dominated by a hilly to undulating landscape of hills and hollows (40 to 75 meters above sea level) [32]. Sub-glacial till, lime-free loam or loamy sand prevail [32]. The topsoil is characterised by soil erosion and a low decalcification depth. Predominant is calcite luvisol/gley luvisol (brown soil family), known for both its fertility and its tendency to compress the topsoil foundation [32]. Typical, indigenous catena sequences are, from the hilltop to hollow, calcarite regosol > eroded calcite luvisol $>$ gley luvisol $>$ eutric gley [33]. In dry years, exposed hilltops are prone to yield reductions, while in wet years the loss occurs on the lower slopes and in the hollows [32]. At the same time, the eroded peaks exhibit higher $\mathrm{pH}$ values than the hollow depressions (Table 1). 
Sandy loam was the predominant soil type in all sub-plots of the pilot facility. Considering a main root zone of $100 \mathrm{~mm}$, sandy loam has a field capacity of $110 \mathrm{~mm}$. In contrast to the hollows, the hilltops are carbonaceous, and average a higher proportion of clay and higher $\mathrm{pH}$ values (Table 1). The carbonate level indicates the initial till substrate which topsoil erosion has brought close to the surface. Soil samples found compressed topsoil foundation (soil depth $20-25 \mathrm{~cm})$ on the shallow calcarite regosol hilltops $(\mathrm{Ah} / \mathrm{C}$ soil). In the samples taken in 2011 , the above-mentioned soil conditions were particularly pronounced. Ploughing was limited on the hilltops due to the shallow topsoil and the initial substrate's proximity to the surface.

\subsection{Climatic Conditions}

The Wilmersdorf climate is characterised by low annual rainfall and frequent dry periods in early summer and autumn. With $517 \mathrm{~mm}$ annual rainfall and an average annual temperature of $8.8^{\circ} \mathrm{C}$, it is one of the driest regions in Germany [32]. The three experiment years (2010-2013) were partially influenced by extreme weather patterns and high annual precipitation that exceeded the long-term average in all three years (Figure 3 ).

In 2010, high precipitation in summer (August: $117 \mathrm{~mm}$; annual rainfall: $640 \mathrm{~mm}$ ), made most fields impassable during both harvest and autumn sowing. 2011 was marked by a very warm and dry April and heavy rains in July (203 mm). Annual rainfall was $693 \mathrm{~mm}$. Winter 2012 brought black frost and a succession of frost/thaw cycles, leading to considerable winterkill. The early sowing test method plots were so badly damaged by winterkill that they had to be reestablished with spring wheat. After a warm and a partially dry spring, above average precipitation fell in July (annual rainfall $626 \mathrm{~mm}$ ). A prolonged and snowy 2012/2013 winter was followed by a pre-summer drought in April, then a dry and warm July and August. With an annual rainfall of $583 \mathrm{~mm}, 2013$ was the driest project year.

\subsection{Test Plots and Implementation}

The field trial was designed as a three-year serial experiment. Complying with crop rotation, the experiments were carried out on a different field each year. Two homogeneous fields (one hilltop, one hollow) were selected on each of these fields by interfacing digital plot, soil and yield maps. The trial cultivation systems were set upon the two sites in fully randomised blocks. Each block contained four plots divided into five sub-plots (20 sub-plots per site/40 sub-plots total). Except for reaping, the $15 \mathrm{~m}$ long by $5 \mathrm{~m}$ wide plots were worked with the customary combine harvester. The individual steps, deadlines and applied techniques are listed in Table 2. The selected ring-cutter tool had a three-meter working width, six cutting rings and a leaf spring roller for reconsolidating the soil. After the winter wheat harvest, the trial plots were completely ploughed and winter rye was cultivated.

Table 1. Soil attributes of the experiment plots.

\begin{tabular}{|c|c|c|c|c|c|c|c|c|c|c|}
\hline Trial No. & Plot No. & $\mathrm{pH}_{K C L}$ & $\mathrm{C}_{\text {org }} \%$ & $\mathrm{CaCO}_{3} \%$ & Gravel \% & Sand \% & Silt \% & Clay\% & Field Cap.\% Vol.+ & Texture class ${ }^{*}$ \\
\hline \multirow[t]{4}{*}{5} & 401-405 & 7.07 & 2.27 & & 4.2 & 60.2 & 29.6 & 10.2 & 18.8 & SaL \\
\hline & $406-410$ & 6.91 & 2.54 & & 2.3 & 60.3 & 28.3 & 11.4 & 19.6 & \\
\hline & 411-415 & 6.95 & 2.5 & & 5.7 & 60.7 & 27.9 & 11.4 & 19.6 & \\
\hline & $416-420$ & 6.99 & 2.89 & & 4 & 56.9 & 29.7 & 13.4 & 21.7 & \\
\hline \multirow[t]{4}{*}{6} & $421-425$ & 7.43 & 3 & & 3.9 & 52.1 & 28.2 & 19.7 & 26.2 & SaL \\
\hline & $426-430$ & 7.4 & 2.82 & 3.51 & 4 & 57.6 & 28 & 14.4 & 22.4 & \\
\hline & $431-435$ & 7.13 & 2.71 & & 1.3 & 59.9 & 28 & 12.1 & 20.3 & \\
\hline & $436-440$ & 7.5 & 3.51 & 2.16 & 1.4 & 52.8 & 26.9 & 20.3 & 26.6 & \\
\hline \multirow[t]{4}{*}{245} & 481-485 & 6.72 & 2.64 & & 3.3 & 55.9 & 33.8 & 10.3 & 20.9 & SaL \\
\hline & $486-490$ & 7.03 & 2.97 & & 1.6 & 54.3 & 33.8 & 11.9 & 22.1 & \\
\hline & 491-495 & 6.01 & 2.39 & & 1.6 & 58.7 & 27.5 & 13.8 & 21.2 & \\
\hline & $496-500$ & 6.69 & 2.84 & & 1.5 & 57 & 31.6 & 11.4 & 20.7 & \\
\hline \multirow[t]{4}{*}{246} & $501-505$ & 7.49 & 2.89 & 7.41 & 1.9 & 53.6 & 31.8 & 14.6 & 23.6 & SaL \\
\hline & $506-510$ & 7.56 & 3.14 & 4.86 & 2.7 & 51.8 & 30.8 & 17.4 & 24.6 & \\
\hline & $511-515$ & 7.73 & 2.65 & 6.97 & 3.1 & 55.2 & 31.6 & 13.2 & 21.8 & \\
\hline & $516-520$ & 7.59 & 2.69 & 7.92 & 4.3 & 54.6 & 29.9 & 15.5 & 22.9 & \\
\hline \multirow[t]{4}{*}{253} & 5101-5105 & 7.32 & 2.39 & & 2.5 & 64.9 & 23.9 & 11.2 & 18.7 & SaL \\
\hline & $5106-5110$ & 7.44 & 2.83 & & 2.7 & 56.8 & 30.5 & 12.7 & 21.5 & \\
\hline & 5111-5115 & 7 & 3.07 & & 1.7 & 58 & 29.9 & 12.1 & 21.7 & \\
\hline & $5116-5120$ & 7.38 & 3.23 & & 1.7 & 55 & 29.1 & 15.9 & 22.1 & \\
\hline \multirow[t]{4}{*}{254} & $5121-5125$ & 7.5 & 3.24 & 4.11 & 2.7 & 56.8 & 29.2 & 14 & 22.4 & SaL \\
\hline & $5126-5130$ & 7.64 & 2.86 & 7.35 & 2.5 & 52.5 & 33.4 & 14.1 & 23.2 & \\
\hline & 5131-5135 & 7.62 & 2.27 & 9.19 & 3.7 & 56.1 & 29.5 & 14.4 & 21.6 & \\
\hline & $5136-5140$ & 7.57 & 2.8 & 4.73 & 2.2 & 60.2 & 26.6 & 13.2 & 21 & \\
\hline
\end{tabular}

\footnotetext{
* estimated with the soli texture triangel USDA; + estimated with the soil water characteristics programm USDA.
} 


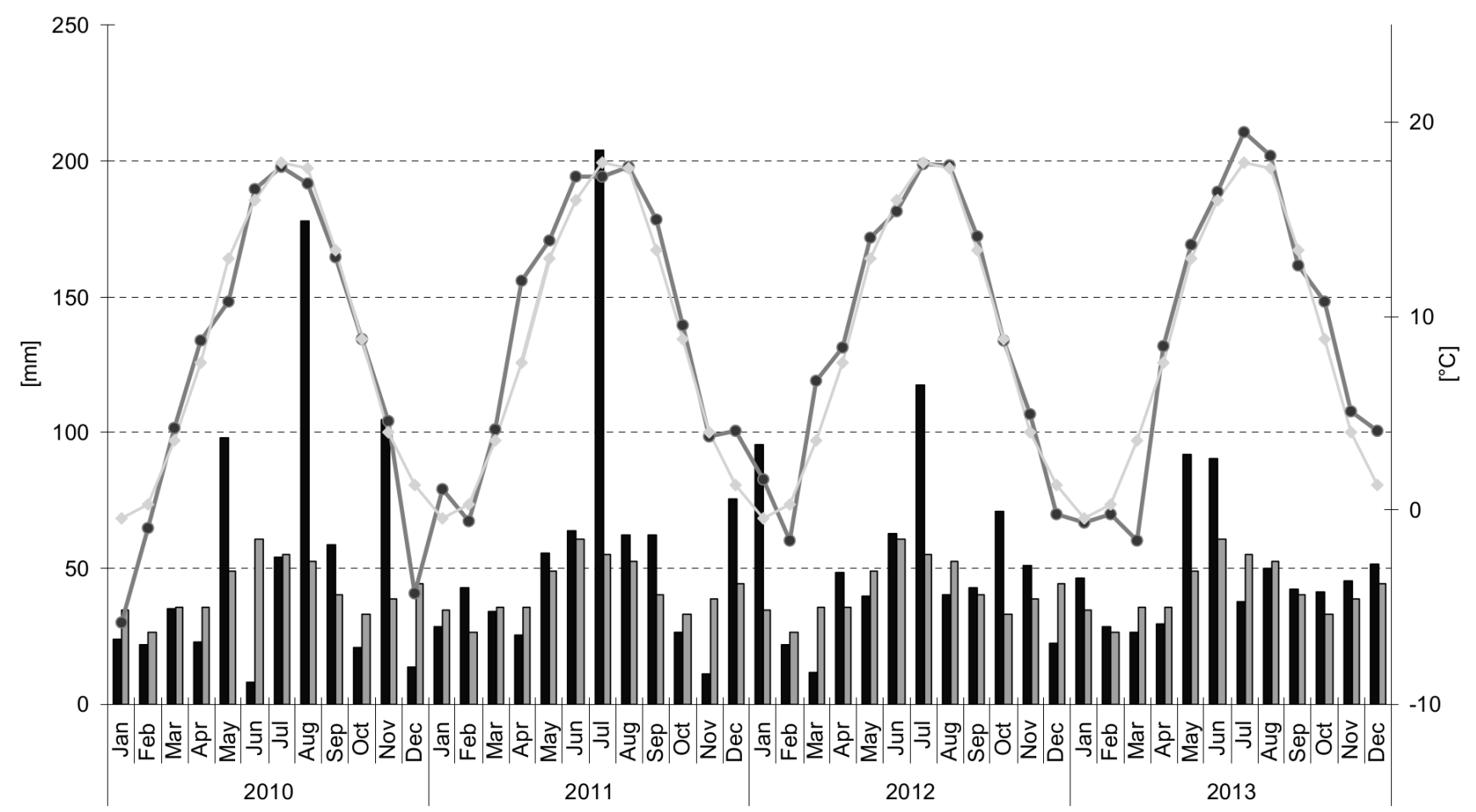

$\square$ Precip. $\square$ Precip. 1971-2000 —Temp. $\multimap$ Temp. 1971-2000

Figure 3. Temperature and precipitation regime at Wilmersdorf project location from 2010 to 2013 (project term) and 1971-2000 (long-term average).

Table 2. Steps and schedule for the Sp (standard practice; plough), ESr (winter wheat early sowing; ring-cutter), CCp (winter wheat following catch crop; plough), Op (winter wheat following oat; plough) and Or (winter wheat following oat; ring-cutter) test methods of winter wheat cultivation following LGS.

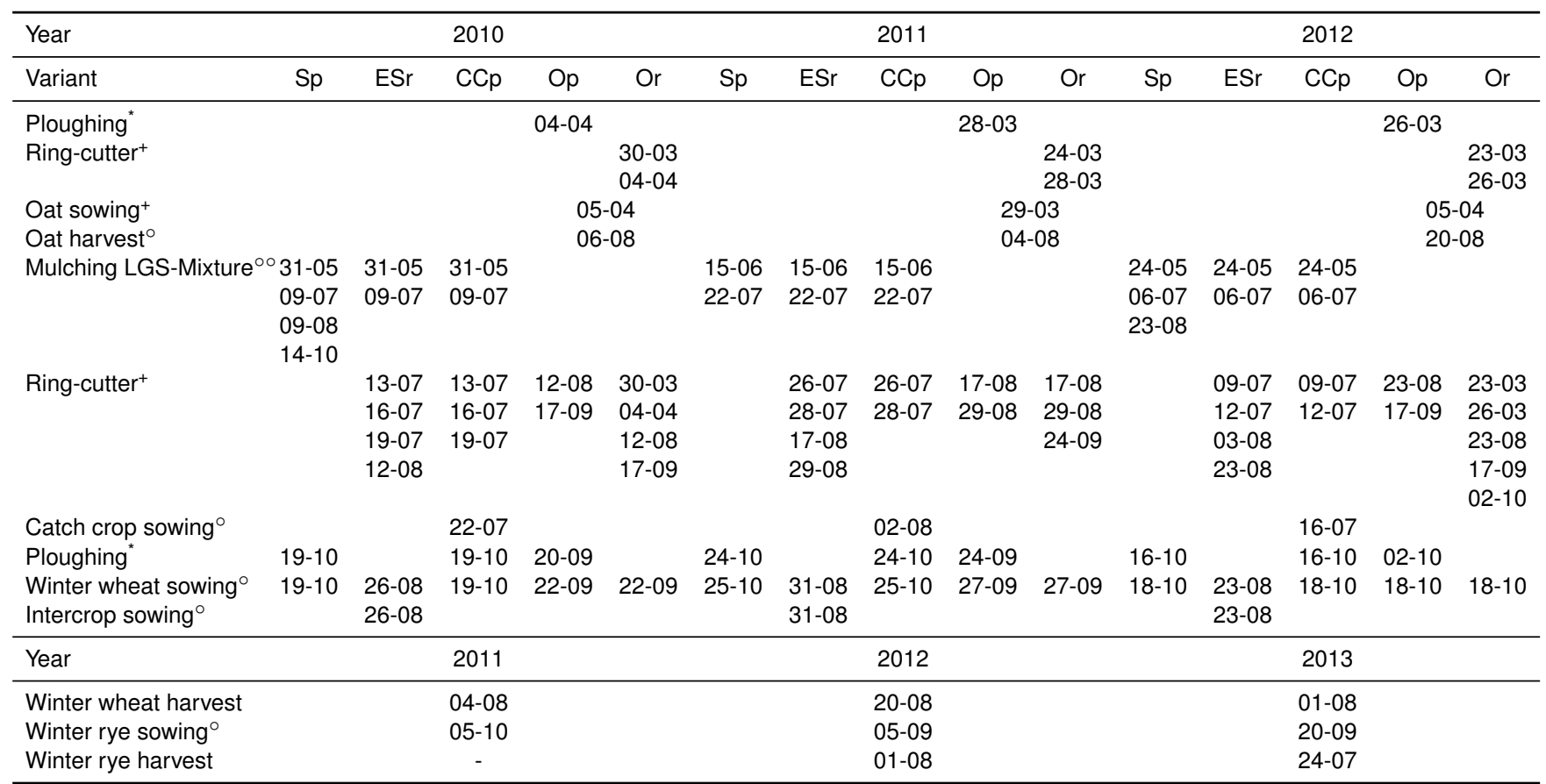

* Lemken Opal 90: Mounted reversible plough with four bodies (working depth $25 \mathrm{~cm}$ );

+ Ring-cutter (working width $3 \mathrm{~m}$; working depth 6-8 cm);

- Väderstad Drill Rapid 300 C (working width $3 \mathrm{~m}$ );

${ }^{\circ}$ Mulching dates depending on the crop height. 


\subsection{Data Compilation and Analysis}

The yields of oat, winter wheat, and subsequent winter rye (2012 and 2013) were assessed by documenting the number of ears per square meter, the thousand kernel weight and the grain yield. The crude protein content in winter wheat, an indication of grain quality, was measured as well as the oat hectolitre weight. The development and structure of crops were determined by visually appraising cultivated plants, weeds and catch crop mixture coverage [34]. Weed appraisal focused on pre-eminent weeds displaying a high degree of both consistency and coverage.

Biomass growth was compiled by taking manual cuts $\left(0.5 \mathrm{~m}^{2}\right)$ from LGS, the oat crop and the summer catch crop. Depending on the test method (Figure 2), two manual LGS cuts were taken from plots of the early sowing and the catch crop method, and three cuts were taken from standard practice plots. All LGS cuts were taken independently, either before the usual mulching dates or prior to using the ring-cutter for the first time, respectively (Table 2). The LGS cuts from oat fields (Zadok scale 69) and from the summer catch crop were taken after buckwheat bloom [8]. To determine the respective yield share, the LGS and oats samples were sorted by hand. The $\mathrm{N}$-input of the second harvest year was determined by calculating LGS biomass yield, leguminosae and $\mathrm{N}$-content. With the $\mathrm{N}$-balance calculator the $\mathrm{N}$-input count was taken from $\mathrm{N}_{2}$-fusion minus gaseous $\mathrm{N}$-losses that arise from mulching [35]. Soil samples to determine Nmin- content were extracted as weather conditions allowed (Pürkhauer drills, soil layer 0-60 cm), i.e. in autumn after winter wheat seeding, at the beginning of vegetation in spring and after the winter wheat harvest. For the extraction of the soil mineral nitrogen, $\mathrm{NH}_{4}-\mathrm{N}$ and $\mathrm{NO}_{3}-\mathrm{N} 2 \mathrm{M} \mathrm{KCl}$ solution was used. In 2010, due to drought, one shallower sample $(0-30 \mathrm{~cm}$ soil layer) was taken after the oat harvest in late summer. The statistical variance analysis was carried out with MIXED procedure (SAS 9.1 statistical software) and subsequently compared to the mean values (Tukey HSD test).

\section{Results}

\subsection{Preceding Crops-Alfalfa-Clover-Grass and Summer Catch Crops}

In accordance with test method design, the same LGS seed mix was sown on all plots over all three years. Over all three years, no substantial differences in the quantity of biomass growth on hilltops and hollows could be discovered (Table 3). However, there was a great difference in growth quality regarding which species grew on which site. On the hilltops, LGS growth regularly exhibited a higher percentage of alfalfa; in the hollows, more grasses, herbs, white clover and weeds (Table 4).

In 2010 and 2011 until mid-October, catch crop test plots achieved growth between 2.5 and 3.9 t DM ha $^{-1}$, ( $\mathrm{N}$ up-take from 72 to $100 \mathrm{~kg} \mathrm{~N} \mathrm{ha}^{-1}$ ). In contrast, in 2012, low rainfall in August and unevenly distributed precipitation in September, caused summer catch crop plots to fail (Table 3).
Table 3. LGS dry mass yields as well as CCp method catch crop dry mass yields (t DM ha ${ }^{-1}$ ). Sp: Standard practice (plough); ESr: Early sowing of winter wheat (ring-cutter); CCp: Winter wheat following catch crop (plough); CC: Catch crop.

\begin{tabular}{|c|c|c|c|c|c|c|}
\hline Site & Year & Test method & Cut 1 & Cut 2 & Cut 3 & $\mathrm{CC}$ \\
\hline \multirow[t]{15}{*}{ Hollow } & 2010 & SP & 4.1 & 3.3 & 2.2 & \\
\hline & & $\mathrm{ESr}$ & 4.7 & 4 & & \\
\hline & & CCp & 4.5 & 3.3 & & 3.7 \\
\hline & & HSD & 2.2 & 0.9 & & \\
\hline & & $p$-value & 0.7179 & 0.0947 & & \\
\hline & 2011 & SP & 5.7 & 5.3 & 4.1 & \\
\hline & & $\mathrm{ESr}$ & 5.4 & 5.9 & & \\
\hline & & CCp & 5.7 & 5.9 & & 2.8 \\
\hline & & HSD & 1.3 & 1.4 & & \\
\hline & & $p$-value & 0.7125 & 0.3542 & & \\
\hline & 2012 & $\mathrm{SP}$ & 5 & 2.4 & 4.4 & \\
\hline & & $\mathrm{ESr}$ & 4.8 & 2.5 & & \\
\hline & & CCp & 5.1 & 2.7 & & \\
\hline & & HSD & 1.4 & 1.1 & & \\
\hline & & p-value & 0.8631 & 0.7744 & & \\
\hline \multirow[t]{15}{*}{ Hill } & 2010 & SP & $4.7^{a}$ & 4.3 & 2.5 & \\
\hline & & $\mathrm{ESr}$ & $4.7^{a}$ & 3.5 & & \\
\hline & & CCp & $5.8^{b}$ & 4.6 & & 3.9 \\
\hline & & HSD & 1 & 2.3 & & \\
\hline & & p-value & 0.0318 & 0.3774 & & \\
\hline & 2011 & SP & 4.9 & 4 & 3 & \\
\hline & & $\mathrm{ESr}$ & 5.2 & 3.6 & & \\
\hline & & CCp & 5.7 & 4.5 & & 2.5 \\
\hline & & HSD & 2.1 & 3.3 & & \\
\hline & & $p$-value & 0.571 & 0.6816 & & \\
\hline & 2012 & SP & 4 & $1.7^{a}$ & 2 & \\
\hline & & $\mathrm{ESr}$ & 4 & $2.3^{a}$ & & \\
\hline & & $\mathrm{CCp}$ & 4.7 & $2.4^{b}$ & & \\
\hline & & HSD & 1.5 & 0.5 & & \\
\hline & & $p$-value & 0.3866 & 0.0067 & & \\
\hline
\end{tabular}

HSD (honestly significant difference);

Different letters indicate significant differences ( $\alpha \leq 0.05)$.

Table 4. Yield shares in \% of alfalfa, white clover, red clover, grass and herbs in the LGS mixture. Alfal: alfalfa; WC: white clover; RC: red clover; herb.: herbs.

\begin{tabular}{llllll}
\hline Site & Year & Alfal. & WC & RC & Grass/herb. \\
\hline Hollow & 2010 & 9 & 66 & & 25 \\
& 2011 & 49 & 4 & & 47 \\
& 2012 & 38 & 15 & & 47 \\
\hline Hill & 2010 & 62 & 23 & & 14 \\
& 2011 & 76 & 1 & & 23 \\
& 2012 & 63 & 5 & 1 & 32 \\
\hline
\end{tabular}

\subsection{Preceding Crop Oats}

In 2010 and 2012, oat yields were 21\% and 54\% higher in the oat/plough test method than in the oat/ring-cutter test method, respectively (Table 5). In each trial year, the yield difference between the two test methods was more pronounced in the hollows than on the peaks. Only on the hilltops in 2011, where ploughing was limited, (see Section 2.2 ), did the unploughed oat ring-cutter method achieve a $17 \%$ higher oat grain yield. 
Table 5. Oat yield structure-Op and Or methods. Op: Oat variant with plough; Or: Oat variant with ring-cutter; tkw: thousand kernel weight.

\begin{tabular}{|c|c|c|c|c|c|c|}
\hline Site & Year & $\begin{array}{l}\text { Test } \\
\text { method }\end{array}$ & $\begin{array}{l}\text { Ears } \\
\left(\mathrm{m}^{2}\right)\end{array}$ & $\begin{array}{l}\text { tkw } \\
(\mathrm{g})\end{array}$ & $\begin{array}{l}\text { Grain yield } \\
\left(\mathrm{t} \mathrm{ha}^{-1}\right)\end{array}$ & $\begin{array}{l}\text { Hectolitre } \\
\text { weight }(\mathrm{kg})\end{array}$ \\
\hline \multirow[t]{12}{*}{ Hollow } & 2010 & Op & 270 & 36.2 & $5.1^{a}$ & 45.8 \\
\hline & & Or & 211 & 35.8 & $2.9^{b}$ & 45.9 \\
\hline & & HSD & 76 & 12 & 1.5 & 0.7 \\
\hline & & $p$-value & 0.0829 & 0.5128 & 0.0214 & 0.1001 \\
\hline & 2011 & Op & 307 & 47.3 & 5.3 & 51.5 \\
\hline & & Or & 280 & 49.1 & 4.6 & 51.4 \\
\hline & & HSD & 41.7 & 4.2 & 2.4 & 2.1 \\
\hline & & $\mathrm{p}$-value & 0.1266 & 0.2744 & 0.4132 & 0.8299 \\
\hline & 2012 & Op & 145 & 41.4 & $3.9^{a}$ & 48.2 \\
\hline & & Or & 178 & 39.9 & $1.8^{b}$ & 48.1 \\
\hline & & HSD & 76 & 2.9 & 0.2 & 3.5 \\
\hline & & $p$-value & 0.2612 & 0.2177 & $<.0001$ & 0.9349 \\
\hline \multirow[t]{12}{*}{ Hill } & 2010 & Op & $243^{a}$ & 36.2 & $4.2^{a}$ & 43.9 \\
\hline & & Or & $181^{b}$ & 36.1 & $3.3^{b}$ & 45.2 \\
\hline & & HSD & 21.4 & 3.2 & 0.4 & 3.8 \\
\hline & & $p$-value & 0.0027 & 0.9624 & 0.0076 & 0.3451 \\
\hline & 2011 & Op & 285 & 46.1 & 3.6 & 49.5 \\
\hline & & Or & 274 & 48 & 4.1 & 50.3 \\
\hline & & HSD & 73.8 & 6.2 & 1.6 & 0.9 \\
\hline & & $p$-value & 0.668 & 0.3883 & 0.3302 & 0.0657 \\
\hline & 2012 & Op & 209 & 41.2 & 4.2 & 47.8 \\
\hline & & Or & 165 & 41.6 & 2.3 & 48.2 \\
\hline & & HSD & 61.8 & 2.3 & 2.3 & 1.6 \\
\hline & & $p$-value & 0.1082 & 0.6804 & 0.0882 & 0.4824 \\
\hline
\end{tabular}

HSD (honestly significant difference);

Different letters indicate significant differences $(\alpha \leq 0.05)$.

\subsection{Winter Wheat}

Among test methods, the winter wheat grain harvest varied considerably. This variance was most pronounced in the years with extreme weather conditions (2011 and 2012; see Section 2.3.).

In 2011 and 2012, winter wheat on the oat/plough and oat-ring-cutter test plots could not be harvested due to frequent heavy precipitation and a rampant weed infestation (Tripleurospermum perforatum). In all years and on all test plots, when harvesting was possible, winter wheat grown with the oat/ring-cutter test method produced the lowest grain yield of all test methods-significantly lower on hilltops in 2012 and 2013; and in hollows in 2013 (Table 6). In addition, the hilltop winter wheat in oat/ring-cutter test methods exhibited decidedly lower crude protein in 2011 and 2012. There was no great difference in grain yields between the standard procedure and early sowing and catch crop test methods. Compared to standard practices, however, the early sowing method had consistently lower grain yields. In 2011, the crude protein content in early sowing was markedly lower than in standard and catch crop methods. In contrast, in the dry year, 2013, early sowing exhibited a much higher thousand kernel weight than standard and catch crop methods. The catch crop method came closest to matching the standard practice in yield and quality. With the exception of catch crop in 2013, none of the alternative test methods were able to achieve a higher yield than the standard practice.

\subsection{Subsequent Crop Winter Rye}

In 2012 and 2013, winter rye was a subsequent crop to winter wheat, bringing in high grain yields, especially in the hollows. With the exception of the thousand kernel weight in the hollows in 2013 there was no discernible difference between the test methods (Table 7).

Table 6. Winter wheat yield and quality. Sp: Standard practice (plough); ESr: Early sowing of winter wheat (ring-cutter); CCp: Winter wheat following catch crop (plough); Op: Winter wheat following oat (plough); Or: Winter wheat following oat (ring-cutter); tkw: thousand kernel weight.

\begin{tabular}{|c|c|c|c|c|c|c|}
\hline Site & Year & $\begin{array}{l}\text { Test } \\
\text { method }\end{array}$ & $\begin{array}{l}\text { Ears } \\
(\mathrm{m} 2)\end{array}$ & $\begin{array}{l}\text { tkw } \\
\text { (g) }\end{array}$ & $\begin{array}{l}\text { Grain yield } \\
\text { (t ha-1) }\end{array}$ & $\begin{array}{l}\text { Crude } \\
\text { protein (\%) }\end{array}$ \\
\hline \multirow[t]{21}{*}{ Hollow } & \multirow[t]{7}{*}{2011} & SP & 256 & 41.9 & 4 & $13.6^{a}$ \\
\hline & & $\mathrm{ESr}$ & 262 & 41.3 & 3.6 & $11.0^{b}$ \\
\hline & & $\mathrm{CCp}$ & 243 & 43.7 & 3.8 & $12.4^{a}$ \\
\hline & & Op & - & - & - & - \\
\hline & & Or & - & - & - & - \\
\hline & & HSD & 84.3 & 3.4 & 2 & 1.3 \\
\hline & & $p$-value & 0.7886 & 0.1727 & 0.807 & 0.0025 \\
\hline & \multirow[t]{7}{*}{2012} & SP & 197 & 46.6 & 3.1 & 13.9 \\
\hline & & $\mathrm{ESr}$ & * & * & * & * \\
\hline & & $\mathrm{CCp}$ & 189 & 47 & 2.9 & 13.9 \\
\hline & & Op & - & - & - & - \\
\hline & & Or & - & - & - & - \\
\hline & & HSD & 127.4 & 5.4 & 2.4 & 1.7 \\
\hline & & $p$-value & 0.8544 & 0.8314 & 0.7934 & 0.9942 \\
\hline & \multirow[t]{7}{*}{2013} & SP & 166 & $43.9^{a}$ & $2.8^{a}$ & 13 \\
\hline & & $\mathrm{ESr}$ & 185 & $48.2^{b}$ & $2.8^{a}$ & 12.6 \\
\hline & & CCp & 191 & $42.9^{a}$ & $2.8^{a}$ & 12.7 \\
\hline & & Op & 187 & $42.2^{a}$ & $2.3^{a}$ & 12.7 \\
\hline & & Or & 181 & $38.4^{c}$ & $1.0^{b}$ & 12.7 \\
\hline & & HSD & 74.1 & 3.3 & 0.8 & 1 \\
\hline & & $p$-value & 0.851 & $<.0001$ & $<.0001$ & 0.7514 \\
\hline \multirow[t]{21}{*}{ Hill } & \multirow[t]{7}{*}{2011} & SP & 270 & $43.9^{a b}$ & $4.6^{a}$ & $12.8^{a}$ \\
\hline & & $\mathrm{ESr}$ & 237 & $41.1^{a}$ & $3.5^{a b}$ & $10.3^{b}$ \\
\hline & & $\mathrm{CCp}$ & 250 & $45.8^{b}$ & $4.5^{a}$ & $11.4^{a}$ \\
\hline & & Op & 176 & $41.8^{a}$ & $3.6^{a b}$ & $9.9^{c}$ \\
\hline & & Or & 158 & $42.5^{a}$ & $1.9^{b}$ & $9.9^{c}$ \\
\hline & & HSD & 157.3 & 2.9 & 2 & 1.5 \\
\hline & & $p$-value & 0.1647 & 0.0018 & 0.0078 & $<.0001$ \\
\hline & \multirow[t]{7}{*}{2012} & SP & $170^{a}$ & 42 & $2.6^{a}$ & $13.6^{a}$ \\
\hline & & $\mathrm{ESr}$ & * & * & * & * \\
\hline & & CCp & $153^{a b}$ & 40 & $2.5^{a b}$ & $13.7^{a}$ \\
\hline & & Op & $93^{b}$ & 40 & $1.3^{a b}$ & $12.6^{a b}$ \\
\hline & & Or & $85^{b}$ & 44.7 & $1.2^{b}$ & $11.6^{b}$ \\
\hline & & HSD & 68.6 & 7.5 & 1.3 & 1.1 \\
\hline & & $p$-value & 0.0082 & 0.359 & 0.0172 & 0.0009 \\
\hline & \multirow[t]{7}{*}{2013} & SP & 218 & $41.7^{a}$ & $2.6^{a b}$ & 11.5 \\
\hline & & $\mathrm{ESr}$ & 177 & $47.1^{c}$ & $2.6^{a b}$ & 11.9 \\
\hline & & $\mathrm{CCp}$ & 188 & $42.6^{a}$ & $2.9^{a}$ & 11.7 \\
\hline & & Op & 234 & $40.5^{a b}$ & $2.2^{b}$ & 11.6 \\
\hline & & Or & 171 & $37.5^{b}$ & $1.2^{c}$ & 11.4 \\
\hline & & HSD & 87 & 3.1 & 0.4 & 0.8 \\
\hline & & $p$-value & 0.1562 & $<.0001$ & $<.0001$ & 0.3644 \\
\hline
\end{tabular}

*Winterkill damage; HSD (honestly significant difference); Different letters indicate significant differences $(\alpha \leq 0.05)$. 
Table 7. Yield structure-subsequent winter rye crop. Sp: Standard practice (plough); ESr: Early sowing of winter wheat (ring cutter); CCp: Winter wheat following catch crop (plough); Op: Winter wheat following oat (plough) and Or: Winter wheat following oat (ring cutter); tkw: thousand kernel weight.

\begin{tabular}{|c|c|c|c|c|c|}
\hline Site & Year & $\begin{array}{l}\text { Test } \\
\text { method }\end{array}$ & $\begin{array}{l}\text { Ears } \\
\left(\mathrm{m}^{2}\right)\end{array}$ & $\begin{array}{l}\text { tkw } \\
(\mathrm{g})\end{array}$ & $\begin{array}{l}\text { Grain } \\
\text { yield }\left(\mathrm{tha}^{-1}\right)\end{array}$ \\
\hline \multirow[t]{14}{*}{ Hollow } & \multirow[t]{7}{*}{2012} & SP & 400 & 34.1 & 6.2 \\
\hline & & ESr & 403 & 33.4 & 6 \\
\hline & & $C C p$ & 351 & 35 & 6.4 \\
\hline & & Op & 370 & 33.9 & 5.9 \\
\hline & & Or & 426 & 35.3 & 6.2 \\
\hline & & HSD & 111.3 & 2.6 & 2.2 \\
\hline & & $p$-value & 0.2842 & 0.2873 & 0.9548 \\
\hline & \multirow[t]{7}{*}{2013} & SP & 438 & $31.9^{a}$ & 4.9 \\
\hline & & $\mathrm{ESr}$ & $438^{*}$ & $34.1^{* a b}$ & $6.0^{*}$ \\
\hline & & $\mathrm{CCp}$ & 485 & $32.2^{a}$ & 5 \\
\hline & & Op & 476 & $32.3^{a}$ & 6.3 \\
\hline & & Or & 430 & $35.4^{b}$ & 5.3 \\
\hline & & HSD & 152.2 & 2.3 & 2.4 \\
\hline & & $\mathrm{p}$-value & 0.6883 & 0.0015 & 0.2964 \\
\hline \multirow[t]{14}{*}{ Hill } & \multirow[t]{7}{*}{2012} & SP & 294 & 35.4 & 5.2 \\
\hline & & $\mathrm{ESr}$ & 357 & 33.4 & 5.1 \\
\hline & & CCp & 306 & 34.7 & 4.8 \\
\hline & & $O p$ & 244 & 34.9 & 4.1 \\
\hline & & Or & 278 & 35.1 & 4.6 \\
\hline & & HSD & 124.9 & 3.2 & 2.2 \\
\hline & & $\mathrm{p}$-value & 0.1238 & 0.3836 & 0.5357 \\
\hline & \multirow[t]{7}{*}{2013} & SP & 244 & 35 & 3.7 \\
\hline & & $\mathrm{ESr}$ & $223^{*}$ & $37.3^{*}$ & $2.9^{*}$ \\
\hline & & $\mathrm{CCp}$ & 269 & 36.8 & 4.2 \\
\hline & & Op & 221 & 36.2 & 2.5 \\
\hline & & Or & 269 & 34.7 & 3.3 \\
\hline & & HSD & 180.37 & 5.3 & 5 \\
\hline & & $\mathrm{p}$-value & 0.8397 & 0.6058 & 0.8433 \\
\hline
\end{tabular}

*Preceding crop summer wheat;

HSD (honestly significant difference);

Different letters indicate significant differences $(\alpha \leq 0.05)$.

\subsection{N-dynamic}

The N-dynamic in cultivation methods was estimated based on the LGS $\mathrm{N}$-input, $\mathrm{N}$-up-take in the grains and $\mathrm{N}_{\text {min }}{ }^{-}$ contents in the soil. Interdependent on tilling date, biomass yield and the leguminous crop, the LGS provided an $\mathrm{N}$-input from 145 to $352 \mathrm{~kg} \mathrm{~N} \mathrm{ha}^{-1}$ in the second harvest year (Table 8). Oats attained the highest nitrogen up-take after LGS tillage and ploughing, taking up more than $100 \mathrm{~kg} \mathrm{~N} \mathrm{ha}^{-1}$ in the hollows in 2010 and 2011.

In late summer 2010 (Table 9), $\mathrm{N}_{\min }$ sampling from the early sowing and catch crop test plots at the hollow exhibited significantly higher $\mathrm{N}_{\min }$ values than the other test methods. Prior to sampling, the LGS plots of both test methods were killed in July via root separation with the ring-cutter (see Table 2).

In the autumn of 2011 and 2012, oat/plough and partly oat/ring-cutter wheat plots exhibited significantly higher $\mathrm{N}_{\min }$-values than those of the standard practice $\left(\varnothing+45 \mathrm{~kg} \mathrm{~N} \mathrm{ha}^{-1}\right.$; Table 10). Oat plough had the highest $\mathrm{N}_{\text {min }}$-values of all test methods. In spring, the highest $\mathrm{N}_{\text {min }}$-values were always observed on standard practice and catch crop wheat plots, which had been ploughed at a later date. Compared to the standard practice, oat/ringcutter and oat/plough methods had significantly lower $\mathrm{N}_{\text {min }}$ values in the spring (Hollow 2011-2013). There were no remarkable differences in $\mathrm{N}_{\text {min }}$-values in samples taken from beneath the subsequent winter rye crop (08.11.2011, 22.10.2012), or taken immediately after the winter wheat harvest (08.02.2013; Table 11). The five highest $\mathrm{N}_{\text {min }}$ values of all sampling dates were found in the subsequent winter rye crop (max. value, $148 \mathrm{~kg} \mathrm{Nha}^{-1}$; oat/ring-cutter test method).

Table 8. $\mathrm{N}$-input and $\mathrm{N}$-uptake in the crop sequence ( $\mathrm{N} \mathrm{kg}$ $\mathrm{ha}^{-1}$ ). Sp: Standard practice (plough); ESr: Early sowing of winter wheat (ring cutter); CCp: Winter wheat following catch crop (plough); Op: Winter wheat following oat (plough); Or: Winter wheat following oat (ring cutter).

\begin{tabular}{|c|c|c|c|c|c|c|c|}
\hline Site & Year & $\begin{array}{l}\text { Test } \\
\text { method }\end{array}$ & $\begin{array}{l}\text { Input } \\
\text { LGS }\end{array}$ & $\begin{array}{l}\text { Uptake } \\
\text { Oat }\end{array}$ & $\begin{array}{l}\text { Uptake } \\
\text { WW }\end{array}$ & $\begin{array}{l}\text { Uptake } \\
\text { WR }\end{array}$ & Balance \\
\hline \multirow[t]{21}{*}{ Hollow } & 2010-2012 & SP & 259 & & 83.8 & 83.7 & 0.32 \\
\hline & & $\mathrm{ESr}$ & 191 & & 59.5 & 78.2 & 0.31 \\
\hline & & CCp & 180 & & 71.2 & 80.3 & 0.4 \\
\hline & & Op & & $105.8^{a}$ & & 74.6 & \\
\hline & & Or & & $56.2^{b}$ & & 76.2 & \\
\hline & & HSD & & 43.7 & 43.5 & 26.5 & \\
\hline & & $p$-value & & 0.0447 & 0.3031 & 0.8273 & \\
\hline & $2011-2013$ & SP & 267 & & 65.4 & 52 & 0.24 \\
\hline & & $\mathrm{ESr}$ & 184 & & & 66 & \\
\hline & & CCp & 211 & & 60.3 & 54.4 & 0.29 \\
\hline & & Op & & 100.4 & & 66.6 & \\
\hline & & Or & & 83 & & 54.4 & \\
\hline & & HSD & & 40.4 & 47.5 & 25 & \\
\hline & & $p$-value & & 0.2642 & 0.7571 & 0.2373 & \\
\hline & 2012-2013 & SP & 206 & & $55.4^{a}$ & & 0.27 \\
\hline & & $\mathrm{ESr}$ & 145 & & $53.2^{a}$ & & 0.37 \\
\hline & & CCp & 146 & & $54.6^{a}$ & & 0.37 \\
\hline & & Op & & $73.2 \mathrm{a}$ & $43.4^{a}$ & & \\
\hline & & Or & & $31.7 \mathrm{~b}$ & $18.8^{b}$ & & \\
\hline & & HSD & & & 14.3 & & \\
\hline & & $\mathrm{p}$-value & & 0.0007 & $<.0001$ & & \\
\hline \multirow[t]{21}{*}{ Hill } & 2010-2012 & SP & 352 & & $87.4^{a}$ & 69.2 & 0.25 \\
\hline & & $\mathrm{ESr}$ & 265 & & $54.2^{b c}$ & 65 & 0.2 \\
\hline & & CCp & 278 & & $77.8^{a b}$ & 60.2 & 0.28 \\
\hline & & Op & & $79.0^{a}$ & $53.8^{b c}$ & 49.5 & \\
\hline & & Or & & $59.0^{b}$ & $29.1^{c}$ & 57.5 & \\
\hline & & HSD & & 10.53 & 32.3 & 27.4 & \\
\hline & & $\mathrm{p}$-value & & 0.0091 & 0.0008 & 0.255 & \\
\hline & 2011-2013 & SP & 222 & & $52.9 a$ & 47.9 & 0.24 \\
\hline & & $\mathrm{ESr}$ & 228 & & & 37.6 & \\
\hline & & CCp & 226 & & $52.3^{a}$ & 55.5 & 0.23 \\
\hline & & Op & & 66.2 & $24.8^{b}$ & 29.7 & \\
\hline & & Or & & 78 & $20.6^{b}$ & 38.5 & \\
\hline & & HSD & & 32 & 25.7 & 64.2 & \\
\hline & & $p$-value & & 0.3229 & 0.0047 & 0.7404 & \\
\hline & 2012-2013 & SP & 149 & & $45.1^{a b}$ & & 0.3 \\
\hline & & $\mathrm{ESr}$ & 156 & & $46.0^{a b}$ & & 0.29 \\
\hline & & CCp & 145 & & $51.3^{a}$ & & 0.35 \\
\hline & & Op & & 69.8 & $37.6^{b}$ & & \\
\hline & & Or & & 39.1 & $20.6^{c}$ & & \\
\hline & & HSD & & 43.4 & 7.4 & & \\
\hline & & $p$-value & & 0.1094 & $<.0001$ & & \\
\hline
\end{tabular}

HSD (honestly significant difference);

Different letters indicate significant differences $(\alpha \leq 0.05)$. 
Table 9. $\mathrm{N}_{\text {min }}$-values of mulched LGS (Sp), of LGS after ring-cutter root separation (ESr), of catch crop mixture (CCp) and of oat stubble (Op und Or)—soil layer 0-30 cm. Sp: Standard practice (plough); ESr: Early sowing of winter wheat (ring-cutter); CCp: Winter wheat following catch crop (plough) Op: Winter wheat following oat (plough); Or: Winter wheat following oat (ring-cutter); HSD (honestly significant difference).

\begin{tabular}{|c|c|c|c|c|c|c|c|c|c|}
\hline \multirow[b]{2}{*}{ Date of sampling } & \multirow[b]{2}{*}{ Site } & \multicolumn{8}{|c|}{$\mathrm{N}_{\min } \mathrm{kg} \mathrm{ha}^{-1}(0-30 \mathrm{~cm})$} \\
\hline & & $\mathrm{Sp}$ & $\mathrm{ESr}$ & CCp & Op & Or & $\bar{x}$ & HSD & p-value \\
\hline \multirow[t]{2}{*}{$11-08-2010$} & Hollow (2010) & $35.8^{b}$ & $109.8^{a}$ & $96.9^{a}$ & $42.4^{b}$ & $22.5^{b}$ & 61.4 & 28.1 & $<.0001$ \\
\hline & Hill (2010) & $34.6^{b}$ & $89.5^{a}$ & $73.5^{a c}$ & $43.0^{b c}$ & $20.0^{b}$ & 52.1 & 32.9 & $<.0001$ \\
\hline
\end{tabular}

Different letters indicate significant differences $(\alpha \leq 0.05)$.

Table 10. $\mathrm{N}_{\min }$-values, (soil layer $0-60 \mathrm{~cm}$ ) beneath winter wheat after autumn seeding and at the onset of spring vegetation. Sp: Standard practice (plough); ESr: Early sowing of winter wheat (ring-cutter); CCp: Winter wheat following catch crop (plough); Op: Winter wheat following oat (plough); Or: Winter wheat following oat (ring-cutter).

\begin{tabular}{llllllllll}
\hline \multirow{2}{*}{ Date of sampling } & \multicolumn{9}{c}{$\mathrm{N}_{\min } \mathrm{kg} \mathrm{ha}^{-1}(0-60 \mathrm{~cm})$} \\
& Site & Sp & ESr & CCp & Op & Or & $\bar{x}$ & HSD & p-value \\
\hline \multirow{2}{*}{$05-04-2011$} & Hollow (2010) & $79.7^{a}$ & $36.7^{b c}$ & $48.6^{c}$ & $25.0^{b}$ & $22.2^{b}$ & 42.4 & 22.4 & $<.0001$ \\
& Hill (2010) & $48.7^{a}$ & $16.9^{b}$ & $36.0^{a}$ & $15.4^{b}$ & $15.6^{b}$ & 26.5 & 15.4 & $<.0001$ \\
& Hollow (2011) & $66.9^{a}$ & $73.9^{a}$ & $77.5^{a}$ & $115.6^{b}$ & $90.3^{a}$ & 84.8 & 39.5 & 0.0153 \\
$24-04-2012$ & Hill (2011) & $49.0^{a}$ & $79.2^{a c}$ & $53.0^{a}$ & $106.6^{c}$ & $98.7^{c}$ & 77.3 & 38.5 & 0.0262 \\
& Hollow (2011) & $99.5^{a}$ & $*^{2}$ & $114.7^{a}$ & $42.9^{b}$ & $42.0^{b}$ & 74.8 & 51.5 & 0.0003 \\
$22-10-2012$ & Hill (2011) & $101.8^{a b}$ & $*$ & $119.3^{a b}$ & $78.3^{a b}$ & $73.7^{b}$ & 93.3 & 55 & 0.0258 \\
& Hollow (2012) & $46.4^{a}$ & $61.1^{a b}$ & $33.7^{a}$ & $77.4^{b}$ & $54.1^{a}$ & 54.5 & 22.8 & 0.0007 \\
$06-03-2013$ & Hill (2012) & $34.0^{a}$ & $48.1^{a b}$ & $39.7^{a}$ & $74.2^{b}$ & $59.0^{a b}$ & 51 & 26.4 & 0.0029 \\
& Hollow (2012) & $76.1^{a}$ & $38.8^{b}$ & $47.3^{b}$ & $38.6^{b}$ & $34.0^{b}$ & 46.9 & 21.7 & 0.0003 \\
& Hill (2012) & 51.6 & 41.5 & 52.6 & 37.3 & 36.2 & 43.8 & 21.8 & 0.0897 \\
\hline
\end{tabular}

*Winterkill damage; Different letters indicate significant differences $(\alpha \leq 0.05)$.

Table 11. $\mathrm{N}_{\min }$-values (soil layer 0-60 cm) under WR subsequent to WW $(08.11 .2011 ; 22.10 .2012)$ and shortly after WW harvest (02.08.2013). Sp: Standard practice (plough); ESr: Early sowing of winter wheat (ring-cutter); CCp: Winter wheat following catch crop (plough); Op: Winter wheat following oat (plough); Or: Winter wheat following oat (ring-cutter); HSD (honestly significant difference).

\begin{tabular}{|c|c|c|c|c|c|c|c|c|c|}
\hline \multirow[b]{2}{*}{ Date of sampling } & \multicolumn{9}{|c|}{$\mathrm{N}_{\min } \mathrm{kg} \mathrm{ha}^{-1}(0-60 \mathrm{~cm})$} \\
\hline & Site & Sp & $\mathrm{ESr}$ & CCp & Op & Or & $\bar{x}$ & HSD & $p$-value \\
\hline \multirow[t]{2}{*}{$08-11-2011$} & Hollow (2010) & 120 & 94.5 & 84.0 & 125.7 & 148.5 & 114.5 & 111.5 & 0.4086 \\
\hline & Hill (2010) & 130.4 & 99.7 & 137.3 & 96.3 & 122.5 & 117.2 & 82.9 & 0.4461 \\
\hline \multirow[t]{2}{*}{$22-10-2012$} & Hollow (2011) & 97 & 70.6 & 87.3 & 50.3 & 66.2 & 74.2 & 64.5 & 0.2293 \\
\hline & Hill (2011) & 104.1 & 121.3 & 116.8 & 66.5 & 97.1 & 101.1 & 75.3 & 0.2184 \\
\hline \multirow[t]{2}{*}{$02-08-2013$} & Hollow (2012) & 41.1 & 35.3 & 40.5 & 38 & 33.4 & 37.6 & 12.3 & 0.2672 \\
\hline & Hill (2012) & 28.5 & 29.9 & 30.2 & 31.1 & 24.6 & 28.8 & 9.8 & 0.306 \\
\hline
\end{tabular}




\subsection{Weeds}

Weed distribution varied on the different sites and test method plots. In all experimental years, the weed population in oats and winter wheat was more pronounced in the hollows than on the hilltops.

On average, the standard practice and catch crop test method (autumn ploughing and late seeding) had the lowest weed dockage, the weed coverage ratio (WCR) in both methods being nearly identical. In comparison, the early sowing WCR was from distinctly to significantly higher in each year. On the oat/ring-cutter plots there was usually a higher WCR (Table 12) and/or a higher weed biomass (Table 13) in established oat and winter wheat cultures than on the oat/plough (Op) test plots. In addition to these quantitative differences, specific differences were found in weed group composition on the varying sites and in the varying test methods.

The prior LGS crops generated weed flora-alfalfa (Medicago sativa $L$.), red and white clover (Trifolium repens $L$. and Trifolium pratense $L$.) as well as perennial ryegrass (Lolium perenne L.) and couch grass (Agropyron repens L.) -on all locations and test methods. Comparing standard practices and ploughed test methods (catch crop, oat/plough) to the unploughed test methods (early sowing and oat/ring-cutter) (Table 13), alfalfa did not regrow in ploughed methods, neither in the preceding oat crop nor or in winter wheat. In 2011, however, alfalfa did regrow on standard practices and catch crop hillop plots because the plough could not optimally turn the soil in autumn. Besides alfalfa, the weed population was dominated by, in descending order, creeping thistle (Cirsium arvense L.), scentless chamomile (Tripleurospermum perforatum), poppy (Papaver rhoeas L.), common chickweed (Stellaria media L.), larkspur (Consolida regalis Gray) and veronica (Veronica chamaedrys L.). These species varied within sites and procedures. In the hollows and in the unploughed test methods, creeping thistle was much more dominant (Table 14), particularly in the oat/ring-cutter winter wheat. In the hollows and on one hilltop, scentless chamomile dominated, mainly in the early sowing, oat/ring-cutter and oat/plough winter wheat test methods (cover ratio, 75-90\%). In 2011 and 2012, a rampant odourless chamomile infestation caused winter wheat crop failure in oat/plough and oat/ring-cutter hollows.

\section{Discussion}

The experimental cultivation methods were carried out from 2010 to 2013 and were at times subject to extreme weather conditions. Only 2013 came close to long-term averages and predicted climate changes, such as pre-summer drought (see Figure 3). With this in mind, the following discussion addresses the extent to which the tests provide a practical alternative to standard practices and if they can contribute to diversifying winter wheat cultivation.

\subsection{Reduced Tillage With the Ring-Cutter}

The hypothesis that the ring-cutter can effectively kill and regulate LGS in both wet (spring) and dry (summer) soils was confirmed-LGS secondary growth coverage on unploughed oat and wheat plots was the same height as on ploughed plots. The tool's cutting technique - a shallow, vertical undercutting of the upper topsoil - separates the alfalfa sprout shaft below the root crowns, effectively impeding regrowth. In contrast to the plough, ring-cutter tractive power requirements are low and operating speed $\left(11 \mathrm{~km} \mathrm{~h}^{-1}\right)$ is remarkably fast. Nonetheless, the tool required two to four working cycles to prepare the plots for mulch seeding after the two-year LGS cultivation. Further drawbacks to the ring-cutter were much lower grain yields in two of the three years on oat/ring-cutter and winter wheat plots and less $\mathrm{N}$-availability than oat/plough plots. Ring-cutter implementation only reached higher oat yields than oat/plough on the shallow hilltop in the 2011 test method. Numerous other studies substantiate that reduced tillage in organic farming results in lower yields and lower N-availability [19,3639]. Reduced tillage limits $\mathrm{N}$-availability by delaying soil organic matter (SOM) mineralisation, due to minimal aeration [19] and poor soil warming in the spring, particularly when densely mulched [40]. Especially in spring, unprocessed earth is often cooler and soggier than ploughed soil, hindering both germination and the initial growth of summer grains [41]. Reduced soil processing can also lead to increased compression in untreated layers $[18,39]$ and to accumulated SOM in the upper soil layer [42], as confirmed by ring-cutter processing research conducted at the ZALF Müncheberg experimental station where much higher soil compression limited root penetration of winter grains [43]. Limited N, compressed soil and thwarted root growth impede a plant's ability to absorb nutrients, which may have caused the much lower oat and winter wheat harvests [37]. In addition to these factors, the ring-cutter plots displayed dense weed growth (Tables 12 and 13); typical for reduced tillage in organic farming $[19,44]$. Shallow ring-cutter processing causes weed seeds to remain close to the surface, providing excellent germination conditions. Perennial species such as couch grass and creeping thistle thrive under reduced tillage [45].

Bearing all this in mind, it becomes clear that the results of reduced tillage are highly dependent on the time of ring-cutter processing, on crop rotation selection and on weather conditions, as confirmed by the completely unploughed oat/ring-cutter and early sowing test methods [18]. While oat/ring-cutter did not generate stable oats or winter wheat grain yields, the early sowing wheat yields of two trial years did not deviate greatly from standard procedure yields. Hence, reduced soil processing can be successful when applied to pertinent sites and adapted to the entire cultivation system, i.e. alterations in crop rotation, seeding and the catch crop [19]. 
Table 12. Percentage weed coverage in all winter wheat cultivation methods (Sp, ESr, CCp Op and Or). Sp: Standard practice (plough); ESr: Early sowing of winter wheat (ring-cutter); CCp: Winter wheat following catch crop (plough); Op: Winter wheat following oat (plough); Or: Winter wheat following oat (ring-cutter); HSD (honestly significant difference).

\begin{tabular}{lllllllllll}
\hline \multirow{2}{*}{ Date } & & \multicolumn{9}{c}{ Weed coverage \% } \\
& Site & Zadoks scale & Sp & ESr & CCp & Op & Or & $\bar{x}$ & HSD & p-value \\
\hline $27-04-2011$ & Hollow (2010) & $30-37$ & $11^{a}$ & $71^{b}$ & $16^{a}$ & $71^{b}$ & $94^{b}$ & 53 & 29.7 & $<.0001$ \\
& Hill (2010) & $32-39$ & $19^{a}$ & $64^{b}$ & $19^{a}$ & $26^{a}$ & $75^{b}$ & 41 & 32.8 & 0.0002 \\
$28-06-2011$ & Hollow (2010) & $77-83$ & $34^{a}$ & $64^{b}$ & $48^{a b}$ & $91^{c}$ & $99^{c}$ & 67 & 25.5 & $<.0001$ \\
& Hill (2010) & $77-83$ & 29 & 50 & 34 & 24 & 50 & 37 & 49.1 & 0.3428 \\
03-05-2012 & Hollow (2011) & $30-34$ & $51^{a}$ & $*$ & $48^{a}$ & $97^{b}$ & $93^{b}$ & 72 & 36.1 & $<.0001$ \\
& Hill (2011) & $30-34$ & $12^{a}$ & $*$ & $11^{a}$ & $8^{a}$ & $54^{b}$ & 21 & 39.4 & 0.0036 \\
$16-07-2012$ & Hollow (2011) & $83-87$ & $73^{a}$ & $*$ & $71^{a}$ & $95^{b}$ & $90^{a b}$ & 83 & 21.5 & 0.0021 \\
& Hill (2011) & $83-87$ & 49 & $*$ & 40 & 45 & 43 & 44 & 42.1 & 0.5208 \\
$23-04-2013$ & Hollow (2012) & $29-31$ & $12^{a}$ & $73^{b}$ & $6^{a}$ & $8^{a}$ & $34^{c}$ & 26 & 15.9 & $<.0001$ \\
& Hill (2012) & $29-31$ & $9^{a c}$ & $71^{b}$ & $5^{a}$ & $6^{a}$ & $21^{c}$ & 22 & 15.4 & $<.0001$ \\
$24-05-2013$ & Hollow (2012) & $41-49$ & $31^{a}$ & $68^{b}$ & $38^{a}$ & $36^{a}$ & $59^{b}$ & 46 & 24.5 & 0.0017 \\
& Hill (2012) & $41-49$ & $20^{a c}$ & $66^{b}$ & $17^{a c}$ & $10^{a}$ & $33^{c}$ & 29 & 21.3 & $<.0001$ \\
\hline
\end{tabular}

*Winterkill damage; Different letters indicate significant differences $(\alpha \leq 0.05)$.

Table 13. Weed biomass in oats (at blossoming), Op and Or methods. Op: Oat (plough); Or: Oat (ring-cutter); HSD (honestly significant difference).

\begin{tabular}{lllllll}
\hline \multirow{2}{*}{ Date } & Site & Op & Or & & HSD & p-value \\
\hline \multirow{2}{*}{ 30-06-2010 } & Hollow (2010) & $1.2^{a}$ & $2.5^{b}$ & 1.8 & 1.2 & 0.0491 \\
& Hill (2010) & 0.7 & 1.6 & 1.2 & 0.7 & 0.1373 \\
09-06-2011 & Hollow (2011) & 0.9 & 1.3 & 1.1 & 1.4 & 0.4211 \\
& Hill (2011) & 0.7 & 0.3 & 0.5 & 0.7 & 0.216 \\
12-06-2012 & Hollow (2012) & 1.8 & 2.2 & 2 & 0.7 & 0.2211 \\
& Hill (2012) & 0.8 & 1 & 0.9 & 1.2 & 0.4896 \\
\hline
\end{tabular}

Different letters indicate significant differences $(\alpha \leq 0.05)$.

Table 14. Dominance (dom.; \%) and frequency (freq.; \%) of Cirsium avense (Ca) and Medicago sativa (Ms) in oat and winter wheat (SP, ESr, CCp, Op and Or test methods). Sp: Standard practice (plough); ESr: Early sowing of winter wheat (ring cutter); CCp: Winter wheat following catch crop (plough); Op: Winter wheat following oat (plough); Or: Winter wheat following oat (ring cutter).

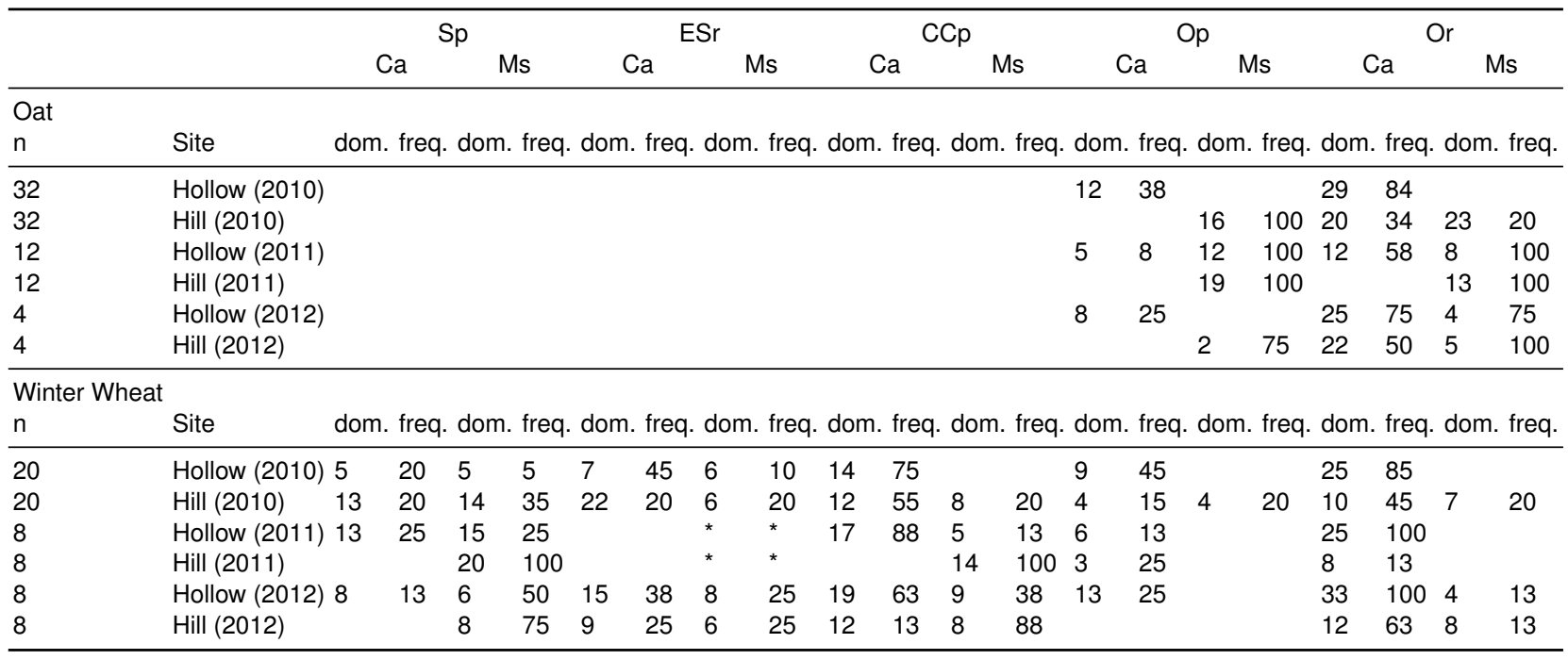




\subsection{Winter Wheat Early Sowing and Summer Catch Crop} Test Methods

In the test methods early sowing and catch crop, LGS processing takes place in the summer. The ring-cutter was used to sever LGS roots by shallowly working the topsoil, even in dehydrated soil (i.e. in June 2010). August 2010 $\mathrm{N}_{\text {min }}$-samples proved that early ring-cutter processing releases significant $\mathrm{N}$-quantities in late summer (up to 109 $\mathrm{kg} \mathrm{N} \mathrm{ha}^{-1}$ ) for early sowing and summer catch crops (see Table 9). Heavy rains in August and November 2010 and in July 2011 caused above average precipitation, possibly leading to nitrate leaching and loss in late summer and autumn, especially in 2010 , in these two test methods. Further indication of such losses is the significantly lower $\mathrm{N}_{\text {min }}{ }^{-}$ value in early sowing and partly in catch crop in spring 2011 , when compared to standard practices.

On the other hand, in 2010 and 2011, catch crop plots developed vigorous cover crops that were able to absorb a portion of the mineralised nitrogen by October (up to 86 $\mathrm{kg} \mathrm{N} \mathrm{ha}^{-1}$ ). Other studies came to similar results with an early catch crop after clover-grass tilling [6]. Compared to standard procedures, the summer catch crop organic material provided a slightly improved $\mathrm{N}$-supply for winter wheat in spring. This is supported by increased $\mathrm{N}_{\text {min }}$-values on catch crop plots in spring 2012. Despite the possible catch crop nitrate transfers, yields were comparable to standard yields, confirming the hypothesis that the catch crop winter wheat test method ploughed in autumn produces equivalent annual grain yields, on hilltops as well as in hollows, where the other test methods reported yield losses. As to location and weather conditions, the catch crop test method proved the hardiest.

Although the early sowing test method yields did not differ greatly from standard practices, compared to catch crop, early sowing yield was characterised by low stability and robustness. In 2012, early sown winter wheat was particularly susceptible to winterkill and in 2011, contained a much lower crude protein. The early sowing test method also had a high weed content despite the catch crop mixture, which is typical in early sown crops [46]. In 2013, with an early drought in April and dry summer months, early sowing came closest to matching standard practice yields. Possibly the more abundant pre-winter growth, followed by a cold and snowy winter in 2012/2013, allowed early sowing winter wheat to exploit the winter moisture and take up nitrogen early, as confirmed by much higher $\mathrm{N}_{\min }$-values on early sowing plots the previous autumn and decidedly lower ones in spring 2013. Due to the developmental advantage of early sowing, winter wheat was perhaps less affected by water shortages in April (stem elongation) and drought in July during the grain-filling phase [25,26]; an idea supported by the significantly higher thousand kernel weight in this year (Table 6). Overall, the early sowing test method shows how heavily grain yields depend on cultivation procedure and weather conditions throughout the year, and that unploughed early sowing is not necessarily synonymous with minimal yield. The only early sowing location effect was weed pressure. This was less pronounced on hilltops and therefore advocates unploughed processing. Based on the grain yield, the early sowing test method is not particularly suitable for dry knoll locations, as was otherwise proposed in hypothesis 3.

\subsection{Winter Wheat Following Oat}

Studies suggesting oat as a good LGS exploiter are confirmed by their results [28]. Especially in the oat/plough test method and in the damp hollows, oats were able to take up high levels of mineralised LGS nitrogen in spring (up to $105 \mathrm{~kg} \mathrm{~N} \mathrm{ha}^{-1}$ ). With a high N-supply, oat/plough strongly suppressed weed growth [29].

Oat/plough and oat/ring-cutter test methods showed high $\mathrm{N}_{m i n}$-values (up to $115.6 \mathrm{~kg} \mathrm{~N} \mathrm{ha}^{-1}$ on oat/plough; $98.7 \mathrm{~kg} \mathrm{~N} \mathrm{ha}^{-1}$ on oat/ring-cutter) at the beginning of November 2011 and the end of October 2012 despite high $\mathrm{N}$-deprivation and an early winter wheat sowing date in 2011. In contrast to standard practice and catch crop, oat/plough and oat/ring-cutter exhibited lower $\mathrm{N}_{\text {min }}$-content in the spring than in autumn, which points to nitrate leaching into deeper soil layers after rainy and cold winter months. In both winters, 2011/2012 (205 mm) and 2012/2013 (149 mm), rainfall from November to February was above field capacity $(110 \mathrm{~mm}$; see Section 2.2.).

Despite summer crop cultivation after springtime LGS tilling, there may be an increased risk of nitrate leaching the following winter [27]. This risk can be offset by establishing a second subsequent crop in early autumn, ensuring a high and rapid N-up-take. Winter wheat N-uptake $\left(10-30 \mathrm{~kg} \mathrm{~N} \mathrm{ha}^{-1}\right)$ in autumn is less pronounced than other grains (for example, winter rye with $30-50 \mathrm{~kg} \mathrm{~N} \mathrm{ha}^{-1}$ ) [47]. Consequently, winter wheat sown on oat/plough and oat/ring-cutter plots at the end of September (2010 and 2011) and in mid-October 2012 only minimally prevented nitrate leaching.

In the spring, oat/plough and oat/ring-cutter winter wheat plots were unable to compete against odourless chamomile. This multiyear species has a fibrous and deepreaching root system, asserting its dominance especially on wet and compacted terrain [48]. Without weed control, odourless chamomile can overrun a winter wheat grain yield by up to $90 \%$ [48]. In the wet years 2011 and 2012, this was irrefutably confirmed in the hollows, locations particularly vulnerable to crop failure [32]. In summary, despite spring tillage and an oat insertion, the nitrogen supply for winter wheat was not improved. Also, the oat/plough and oat/ringcutter test methods were highly susceptible to weeds. Only on the hilltops and in the dry year 2013 was oat/plough winter wheat yield comparable to standard practices, contrary to hypothesis 2 . In each trial year and compared to standard practice, the oat/ring-cutter winter wheat hilltop harvests produced a smaller deficit margin than harvests in the hollows did. According to hypothesis 3, the oat method is more suitable for hilltops. However, it is not a viable al- 
ternative to standard cultivation practices as its yield levels are much lower.

\section{Conclusion and Outlook}

When it comes to adapting cultivation methods to climate changes, greater diversity is an effective risk management strategy for agricultural enterprises [12]. Practically applied, cultivation and soil processing methods must nurture crop robustness. The unpredictable influences of our changing climate demand crops that are insensitive to a broad spectrum of influences [15]. The field test cultivation methods revealed distinctly variable degrees of robustness when it came to growth sites and weather conditions. In every field test year, the standard practice and the catch crop test method produced relatively hardy grain and stable crops on all plots. Waiving LGS virgin tilling in autumn as well as improving work-cycle peaks make catch crop a viable alternative, contributing to winter wheat diversification. Assuming that future climate changes lead to less winter frosts and therefore less risk of winterkill, the early sowing test method could be an equally feasible option for winter wheat diversification [5]. This is clearly shown in early sowing yield levels which were comparable to standard practice yields during the dry year 2013. The oat/plough and oat/ringcutter test methods were far more precarious than standard practices or the early sowing and catch crop test methods. Both oat test methods are highly site and weather sensitive, which resulted in two years of failed winter wheat crops in the soggy hollows. Assuming that climate change brings an increase in heavy rainfall [5], the weaknesses of oat/ringcutter and oat/plough (saturated hollows and rampant weed infestation) make this test method impracticable.

Ring-cutter processing proved to be overall practical, and particularly suited to organic farming needs (multipleyear LGS tillage). However, the denser weed growth, lower $\mathrm{N}$ mineralisation and lower grain yields, clearly proves it

\section{References}

[1] Milestad R, Dedieu B, Darnhofer I, Bellon S. Farm and farmers facing change: The adaptive approach. In: Darnhofer I, Gibbon D, Dedieu B, editors. Farming Systems Research into the 21st Century: The New Dynamic. Dordrecht: Springer Netherlands; 2012. pp. 365-385.

[2] Bloch R, Knierim A, Häring AM, Bachinger J. Increasing the adaptive capacity of organic farming systems in the face of climate change using action research methods. Organic Agriculture. 2015; Available from: http://dx.doi.org/10.1007/s13165-015-0123-5.

[3] Pang XP, Letey J. Organic Farming Challenge of Timing Nitrogen Availability to Crop Nitrogen Requirements. Soil Science Society of America Journal. 2000;64:247-253. cannot replace the plough. Nonetheless, as a flexible, manageable tool, the ring-cutter is indeed an alternative on fields where site or weather conditions create severe ploughing risks, such as ploughing depth compaction and hilltop erosion. Climate changes are also changing the customary ploughing and/or soil processing dates [4]. The ring-cutter can help to increase the number of fieldwork days, allowing for LGS processing on both wet and dry soil. The field tests made evident, however, that reduced ploughing must go hand in hand with adjusting cultivation methods and scheduling. The ploughing in spring and autumn resulted in severely reduced oat and winter wheat yields, which was not the case when ploughing in summer. This confirms observations in organic farming that timing is often more decisive than method when it comes to $\mathrm{N}$-supply and weed regulation [49]. If an appropriate moment is not seized, the ensuing consequences cannot be offset by turning to shortterm resources, as is the case in conventional farming [9]. This is a distinctive issue and climate change adaptation is a unique and demanding process for organic farmers [2]. There are no universally applicable measures for increasing diversification and robustness on organic farms. A farm's individual site and operating needs must be taken into account to bring robustness to the entire system [50]. In order to identify and develop specific measures for adapting organic farms to climate changes, on-site projects such as this one are indispensable.

\section{Acknowledgments}

This work was funded by the Federal Ministry of Education and Research (BMBF), Germany, by the Federal Ministry of Food, Agriculture and Consumer Protection (BMELV), Germany and by the Ministry of Science, Research and Culture (MWFK), Brandenburg. We thank Mr. Stefan Palme, organic farmer, for his expert advice and for allowing the experiments to be conducted on Wilmersdorf.

[4] Reyer C, Bachinger J, Bloch R, Hattermann FF, Ibisch PL, Kreft S, et al. Climate change adaptation and sustainable regional development: a case study for the Federal State of Brandenburg, Germany. Regional Environmental Change. 2012;12(3):523-542.

[5] Gerstengarbe FW, Badeck F, Hattermann F, Krysanova V, Lahmer W, Lasch P, et al.. Gerstengarbe FW, editor. Studie zur klimatischen Entwicklung im Land Brandenburg bis 2055 und deren Auswirkungen auf den Wasserhaushalt, die Forst- und Landwirtschaft sowie die Ableitung erster Perspektiven. Potsdam;.

[6] Heß J. Kleegrasumbruch im Organischen Landbau: Stickstoffdynamik im Fruchtfolgeglied Kleegras - Kleegras - Weizen - Roggen [Dissertation]. Rheinische Friedrich-Wilhelms-Universität Bonn, Department of Agronomy. Bonn, Germany; 1989.

[7] Stanford, G, Epstein E. Nitrogen Mineralization-Water 
Relations in Soils. Soil Science Society of America. 1974;38:103-107.

[8] Zadoks JC, Chang TT, Konzak CF. A Decimal Code for the Growth Stages of Cereals. Weed Research. 1974;14:415-421.

[9] Eitzinger J, Kersebaum KC, Formayer H, editors. Landwirtschaft im Klimawandel: Auswirkungen und Anpassungsstrategien für die Land- und Forstwirtschaft in Mitteleuropa. 1st ed. Clenze, Germany: Agrimedia; 2009. Available from: http://www.gbv.de/dms/zbw/600031977.pdf/http://de. agrimedia.com/libeconomy/shop/detail.php?id=268.

[10] Trnka M, Olesen JE, Kersebaum KC, Skjelvåg $A O$, Eitzinger J, Seguin B, et al. Agroclimatic conditions in Europe under climate change. Global Change Biology. 2011;17(7):2298-2318.

[11] Bloch R, Wechsung F, Heß J, Bachinger J. Climate change impacts of legume-grass swards: implications for organic farming in the Federal State of Brandenburg, Germany. Regional Environmental Change. 2015;15(2):405-414.

[12] Lin BB. Resilience in Agriculture through Crop Diversification: Adaptive Management for Environmental Change. BioScience. 2011;61(3):183-193.

[13] Smit B, Skinner M. Adaptation Options in Agriculture to Climate Change: a typology. Mitigation and Adaptation Strategies for Global Change. 2002;7:85-114.

[14] Hallegate S. Strategies to adapt to an uncertain climate change. Global Environmental Change. 2009;19:240-247.

[15] Birkmann J, Böhm HR, Buchholz F, Büscher D, Daschkeit A, Ebert S, et al. Glossar Klimawandel und Raumentwicklung: 2. überarbeitete Fassung. E-Paper der ARL. 2013;(10).

[16] Askegaard M, Olesen JE, Rasmussen IA, Kristensen $\mathrm{K}$. Nitrate leaching from organic arable crop rotations is mostly determined by autumn field management. Agriculture, Ecosystems and Environment. 2011;142:149160.

[17] McMillen M. The Effect of Mulch Type and Thickness on the Soil Surface Evaporation Rate. Horticulture and Crop Science. 2013;Available from: http: //digitalcommons.calpoly.edu/hcssp/22.

[18] Tebrügge F, Düring RA. Reducing tillage intensity - a review of results from a long-term study in Germany. Soil \& Tillage Research. 1999;53:15-28.

[19] Peigné J, Ball BC, Roger-Estrade J, David C. Is conservation tillage suitable for organic farming? A review. Soil Use and Management. 2007;23:129-144.

[20] Kladivko EJ. Tillage systems and soil ecology. Soil \& Tillage Research. 2001;61:61-76.

[21] Holland JM. The environmental consequences of adopting conservation tillage in Europe: reviewing the evidence. Agriculture, Ecosystems \& Environment. 2004;103:1-25.

[22] Myrbeck A, Stenberg M, Rydberg T. Establishment of winter wheat-Strategies for reducing the risk of nitrogen leaching in a cool-temperate region. Soil \& Tillage Research. 2012;120:25-31.

[23] Thomsen IK, Lægdsmand M, Olesen JE. Crop growth and nitrogen turnover under increased temperatures and low autumn and winter light intensity. Agriculture, Ecosystems and Environment. 2010;139((1-2)):187194.

[24] Liebman M, Dyck E. Crop Rotation and Intercropping Strategies for Weed Management. Ecological Applications. 1993;3(1):92-122.

[25] Nendel C, Kersebaum KC, Mirschel W, Wenkel KO. Testing farm management options as climate change adaptation strategies usingthe MONICA model. European Journal of Agronomy. 2012;52:47-56.

[26] Olesen JE, Trnka M, Kersebaum KC, Skjelvåg AO, Seguin B, Peltonen-Sainio P, et al. Impacts and adaptation of European crop production systems to climate change. European Journal of Agronomy. 2011;34:96112.

[27] Berry PM, Sylvester-Bradley R, Philipps L, Hatch DJ, Cuttle SP, Rayns FW, et al. Is the productivity of organic farms restricted by the supply of available nitrogen? Soil Use Management. 2002;18:248-255.

[28] Watson CA, Baddeley JA, Edwards AC, Rees RM, Walker RL, Topp CFE. Influence of ley duration on the yield and quality of the subsequent cereal crop (spring oats) in an organically managed long-term crop rotation experiment. Organic Agriculture. 2011;1(3):147159.

[29] Seavers GP, Wright KJ. Crop canopy development and structure influence weed suppression. Weed Research. 1999;39(4):319-328.

[30] Lemanczyk G, Sadowski CK. Fungal communities and health status of roots of winter wheat cultivated after oats and oats mixed with other crops. BioControl. 2002;47(3):349-361.

[31] Kaukoranta T, Hakala K. Impact of spring warming on sowing times of cereal, potato and sugar beet in Finland. Agricultural and Food Science. 2008;17:165176.

[32] Schmidt R. In: Flade M, editor. Naturräumliche Gliederung von Landschaften. Wiebelsheim, Germany: Quelle \& Meyer; 2003. pp. 40-45.

[33] Wirth SJ. Soil Microbial Properties Across an Encatchment in the Moraine, Agricultural Landscape of Northeast Germany. Geomicrobiology Journal. 1999;16(3):207-219.

[34] Wilbois KP, Schwab A, Fischer H, Bachinger J, Palme $\mathrm{S}$, Petersen $\mathrm{H}$, et al. Leitfaden für Praxisversuch: Eine Anleitung zur Planung, Durchführung und Auswertung von Praxisversuchen. Kooperationsgemeinschaft: FiBL Deutschland e.V., ZALF e.V. and Gut Wilmersdorf GbR; 2004. Available from: http://www.praxisversuche. de/index.php.

[35] Bachinger J, Reckling M, Stein-Bachinger K. N-budget calculator for legume grass-mixtures. Leibniz-Centre for Agricultural Landscape Research; 2013. Available 
from: http://www.zalf.de/de/forschung/institute/lse/ downloads/Documents/oekolandbau/saldorechner/ Nbudget_calculator.xIs.

[36] Peigné J, Messmer M, Aveline A, Berner A, Mäder $P$, Carcea $M$, et al. Wheat yield and quality as influenced by reduced tillage in organic farming. Organic Agriculture. 2014;4(1):1-13.

[37] Vakali C, Zaller JG, Köpke U. Reduced tillage effects on soil properties and growth of cereals and associated weeds under organic farming. Soil \& Tillage Research. 2011;111:133-141.

[38] Berner A, Hildermann I, Fließbach A, Pfiffner L, Mäder P. Crop yield and soil fertility response to reduced tillage under organic management. Soil \& Tillage Research. 2008;101:89-96.

[39] Emmerling C. Reduced and Conservation Tillage Effects on Soil Ecological Properties in an Organic Farming System. Biological Agriculture and Horticulture. 2007;24:363-377.

[40] Triplett Jr GB, Dick WA. No-Tillage Crop Production: A Revolution in Agriculture! Agronomy Journal. 2008;100:153-165.

[41] Gruber S, Möhring J, Claupein W. On the way towards conservation tillage-soil moisture and mineral nitrogen in a long-term field experiment in Germany. Soil \& Tillage Research. 2011;115-116:80-89.

[42] Hamza M, Anderson W. Soil compaction in cropping systems. A review of the nature, causes and possible solutions. Soil \& Tillage Research. 2005;82:121-145.

[43] Hofbauer M, Bloch R, Gerke HH, Bachinger J. Effekte des Ringschneidereinsatzes zur pfluglosen Bodenbearbeitung auf physikalische Eigenschaften sandiger Böden. In: Neuhoff D, Stumm C, Ziegler S, Rahmann G, Hamm U, Köpke U, editors. Beiträge zur 12. Wissenschaftstagung Ökologischer Landbau. Berlin, Germany: Köster Berlin; 2013. pp. 34-37.

[44] Mäder P, Berner A. Development of reduced tillage systems in organic farming in Europe. Renewable Agriculture and Food Systems. 2012;27(01):7-11.

[45] Bàrberi, P. Weed management in organic agriculture: are we addressing the right issues? Weed Research. 2002;42:177-193.

[46] Kolb LN, Gallandt ER. Weed management in organic cereals: advances and opportunities. Organic Agriculture. 2012;2(1):23-42.

[47] SMUL. Sächsische Landesanstalt für Landwirtschaft, editor. Umsetzung der Düngeverordnung: Hinweise und Richtwerte für die Praxis. Dresden; 2007. Available from: https://publikationen.sachsen.de/bdb/ artikel/15242.

[48] Kay QON. Tripleurospermum Inodorum (L.) Schultz Bip. Journal of Ecology. 1994;82(3):681-697.

[49] Schmid H. Bodenbearbeitung im ökologischen Ackerbau ohne tiefes Pflügen. Justus-Liebig-Universität Gießen; 2011.

[50] Gattinger A, Fliessbach A. Rolle des Ökologischen Landbaus beim Klimawandel. In: Agrarsoziale Gesellschaft e V (ASG), editor. Klimaschutz- und Anpassungsstrategien in Landwirtschaft und ländlichem Raum. vol. 61. Göttingen; 2010. 


\title{
An Experimental Test of a Biodynamic Method of Weed Suppression: The Biodynamic Seed Peppers
}

\author{
Bruce K. Kirchoff
}

Department of Biology, University of North Carolina at Greensboro Greensboro, NC, USA. E-Mail: kircohff@uncg.edu; Tel.: +1 3363344953; Fax: +1 3363345839

Submitted: 16 February 2016 | In revised form: 14 April 2016 | Accepted: 15 April 2016 | Published: 27 April 2016

\begin{abstract}
An experimental test of a biodynamic agriculture method of weed suppression was carried out in growth chambers to establish the feasibility of the method as a preliminary to field trials. Four generations of Brassica rapa plants were used in a randomized block design. Treated flats received ashed seeds prepared according to biodynamic indications. Seed weight and counts were measured at the end of each generation, and germination of the control and experimental seed was investigated at the end of generation four. The biodynamic seed peppers, created and applied as described here, had no effect on seed production or viability, and did not effectively inhibit reproduction of the targeted species over the course of four consecutive treatments.
\end{abstract}

Keywords: Biodynamics; biodynamic agriculture; weeds; invasive plants

\section{Introduction}

Invasive plants (weeds) create serious ecological problems in both agricultural and natural systems. With each introduction the risks of detrimental effects increase. The intruders usually lack natural enemies to control their proliferation, often grow quickly, and can become major pests [1]. These conditions have necessitated the development of methods for removing or controlling the invasive plant. Unfortunately, invasive species control is costly, often involves the use of chemicals, and is often not appropriate for use in natural areas $[2,3]$. Under these conditions it is worthwhile to investigate alternative methods of invasive plant control, even if they are unconventional.

There are five currently accepted methods of weed control [3]: mechanical removal or destruction; prescribed fire; grazing; biological control; and the use of herbicides. These methods can be used alone or in combination, and are intended to produce a maximum effect on a targeted weed while minimizing harmful effects to the landscape. All but the last of these are acceptable organic practices. Unfortunately, all five approaches contain some risk of damaging habitats in which they are used, and all lack complete efficacy.

The damage caused by invasive species is massive. The annual damage has been estimated at U\$33 billion, out of a total crop agricultural production of approximately U\$267 billion in the United States [4]. Clearly, safe, efficient and cost effective methods for controlling or eradicating invasive species are badly needed.

In 1924 the founder of the first organic system of agriculture, Rudolf Steiner, proposed a method of weed control as part of his system of biodynamic agriculture [5]. This method uses an ash prepared from the seeds of the weed that is to be controlled, a "pepper", that is spread over the affected area. Steiner asserted that this treatment, when properly prepared to take advantage of the forces of the moon, will eradicate the treated species after four years 
of applications. Although there are numerous anecdotal reports on the efficacy of this method [6] (Grant Lyon, Jon Lyerly, Hugh Courtney, personal communications), there have been no peer-reviewed tests of its efficacy.

Steiner's method for controlling a weed infestation is relatively simple. One collects the seeds of the weed and burns them over a wood fire at the proper moon phase. The resulting ash is then scattered over the affected area. Steiner claims that after two years of treatments there will be a noticeable reduction in the population of the weed. After four years of treatments the weed will cease to inhabit the treated area [5]. In order to test these claims we investigated Steiner's method of weed control under controlled growth chamber conditions, and measured its effects on seed production and seed viability over four generations.

\section{Methods and Materials}

Four generations of Brassica rapa L. plants [7] were grown under continuous light at $22^{\circ} \mathrm{C}$ in controlled environmental chambers (Environmental Growth Chambers, model: GCW15) at the University of North Carolina at Greensboro. Brassica rapa was chosen because it has a life cycle of approximately 45 days under continuous light, and because its seeds are retained within the fruit at maturity. Many weeds spontaneously release their seeds making it difficult or impossible to determine the reproductive output of the plant. Seeds were obtained from Carolina Biological Supply Company (Wisconsin Fast Plants, standard rapid cycling), and the Rapid Cycling Brassica Collection in Maddison, WI (www.fastplants.org; standard rapid cycling, $\mathrm{RCI}$ ). We used four consecutive generations of the experiment to simulate the four years Steiner claimed would be effective in eradicating the weed.

Following Steiner's indications, we created the seed pepper by burning the seeds over a wood fire within 24 hours of the full moon (i.e., during the early waning moon) [5]. As the pepper was mixed with wood ash as a result of the combustion process, a second wood fire with no added seeds was used to create control replicates of untreated ash. The two fires were burned side by side, at the same time. The ashed seeds and wood ash were crushed to a powder using a mortar and pestle, and the resulting pepper and control ash were weighed and divided into equal packets to be spread over the flats.

The experimental apparatus consisted of twenty perforated half-flats $(35.6 \times 35.6 \mathrm{~cm}), 10$ for the control group and 10 for the experimental group. Each flat was filled with approximately $3 \mathrm{~L}$ of Fafard $3 \mathrm{~b}$ soil mix (Conrad Fafard Inc., U.S.). Thirty seeds were planted in each flat, two per hole, approximately $3 \mathrm{~mm}$ below the soil surface.

Each flat was randomly assigned to either the control or treatment group. The 10 experimental flats were dusted with equal amounts of the seed pepper at the beginning of each generation, while the 10 control flats were treated with equal amounts of wood ash at the same time. All 20 flats were placed in growth chambers in a randomized block design.
Soon after germination each flat was thinned to contain only fifteen seedlings, one per hole. For the length of the experiment the flats were watered through a reservoir system. Each flat received $1 \mathrm{~L}$ of water every other day. In order to assure continued plant health in the nutrient poor soil mix, generations 3 and 4 were fertilized once a week with Peter's 20-20-20 general purpose water soluble fertilizer (Scott's Co. LLC., U.S.). The fertilizer was mixed at a dilution of 0.24 $\mathrm{L}$ of fertilizer to $94.6 \mathrm{~L}$ of water. One liter of the fertilizer mixture was substituted for water every 7 days.

Brassica rapa requires cross pollination to set seed [8]. The pollen is sticky, and not easily susceptible to being carried by the wind [8]. Under field conditions outcrossing is accomplished primarily by physical contact between neighboring plants, presumably due to plant swaying [8]. In our growth chambers the flats were placed approximately 15 $\mathrm{cm}$ apart to minimize contact between plants in different flats. Pollination was accomplished by using bee-sticks, dehydrated bee thoraxes glued to the ends of small sticks (www.fastplants.org). Individual bee-sticks were assembled for each flat, and used only for pollinating within that flat. This restricted pollen flow to each flat. Plants were pollinated over a five day period beginning on day 17 of each growth period.

On day 35, watering was discontinued and the plants were left undisturbed for one week to allow for the maturation of the seeds. At the end of the maturation period, seeds from each flat were harvested and kept separate. Seeds produced from a particular flat were used to plant the next generation of the experiment in that same flat.

For all but the second generation, the seeds from each half-flat were weighed in aggregate. In generation two, which produced few seeds, the number of seeds per flat was counted directly.

To assess differences in mean seed production in each generation, one-tailed t-tests under the assumption of equal variances were performed with SPSS version 19 or 22 $[9,10]$. One-tailed tests were used because Steiner's hypothesis predicts lower seed numbers/weights following treatment with the seed peppers. The t-tests for generations one, three and four compared mean seed weights per treatment, while in generation two the number of seeds produced between the control and experimental groups was compared.

We also checked for differences in percent germination between the control and experimental groups using the seeds produced from the final generation.

\section{Results}

There was no significant difference between seed set in the control and experimental groups in any of the four generations (Table 1). Germination rates were not significantly different between seeds of the two treatments after four generations (Table 2). In all cases, Levene's Test for Equality of Variances was not significant. In generations two and four the control group produced fewer seeds than the experimental. 
Table 1. Four generations biodynamic seed pepper treatments.

\begin{tabular}{llllll}
\hline Gen. & Treatment & $\begin{array}{l}\text { Seed number } \\
(\text { mean } \pm \mathrm{SE})\end{array}$ & $\begin{array}{l}\text { Seed weight } \\
(\mathrm{g} ; \text { mean } \pm \mathrm{SE})\end{array}$ & $\mathrm{p}$-value & $95 \% \mathrm{Cl}$ \\
\hline 1 & Control & & $2.44 \pm 0.44$ & 0.46 & $-0.44 ; 0.49$ \\
1 & Experimental & & $2.42 \pm 0.55$ & & \\
2 & Control & $43.9 \pm 8.5$ & & 0.46 & $-50.85 ; 46.05$ \\
2 & Experimental & $46.3 \pm 21.5$ & & & \\
3 & Control & & $11.0 \pm 2.0$ & 0.23 & $-3.14 ; 6.7$ \\
3 & Experimental & $9.22 \pm 1.22$ & & \\
4 & Control & $2.08 \pm 0.20$ & 0.15 & $-0.81 ; 0.26$ \\
4 & Experimental & & $2.35 \pm 0.15$ & & \\
\hline \multicolumn{5}{l}{ (Gen.: generation; SE: standard error; Cl: confidence interval) }
\end{tabular}

Table 2. Germination rates after four generations.

\begin{tabular}{lll}
\hline Treatment & $\begin{array}{l}\text { Number seeds germinated } \\
( \pm \text { SE; out of } 30)\end{array}$ & $p$-value \\
\hline Control & $22.70 \pm 1.34$ & 0.11 \\
Experimental & $24.70 \pm 0.86$ & \\
\hline
\end{tabular}

(SE: standard error)

\section{Discussion}

Under the conditions detailed here we observed no decrease in seed viability or production in $B$. rapa with treatment with a biodynamic seed pepper.

We attribute the low seed production of generation two to the exhaustion of nutrients from the already nutrient-poor potting soil. The addition of fertilizer at the beginning of generation three restored normal yields.

The fact that the control group in generation four showed lower seed production ( $2.08 \mathrm{~g}$ versus $2.35 \mathrm{~g}$ ), and had a lower germination rate (22.7/30 versus $24.7 / 30$ seeds) than the experimental group is contrary to Steiner's prediction, though these results are not statistically significant.

Prior to carrying out this work, we performed preliminary experiments to test the effect of Biodynamic seed peppers on seed germination of okra seeds (Abelmoschus esculentus L. Moench) $[11,12]$. These experiments yielded negative results after three generations of treatments. An experiment testing the effects of a biodynamic pepper prepared from the burned skin of the brushtail possum (Trichosurus vulpecula Kerr) in New Zealand also gave negative results [13]. Similar negative results were obtained by Bächi-Kunz using a BD pepper to control the Red Flower Beetle (Tribolium castaneum Herbst) [14].

The most extensive series of experiments with the biodynamic seed peppers was performed in Germany over a seventeen year period, but the results were never published outside a little known conference proceeding [15], which was unknown to us until after we completed our experiments. Spieß tested the seed peppers on dandelions (Taraxacum officinale F.H. Wigg.) and other agricultural weeds growing in open fields, and in a variety of containers. The effects of the peppers on seed germination were also investigated. Biodynamic agricultural practices were used throughout these trials. In the field experiments, various preparations of the peppers were spread annually on the experimental fields, while no treatment was applied to the control fields. Three separate types of ash preparations were used on separate experimental plots: ash prepared at the full moon, ash prepared at the new moon, and ash prepared to a homeopathic potency of D8 following the suggestion of Thun [16]. Results of the treatments were measured as the number of dandelion inflorescences produced per square meter (Figure 1). Negative results were observed during the first four years, in which the number of inflorescences in the control group was consistently less than those of the experimental group. In the remaining thirteen years, Spieß observed only four years in which the control groups showed a higher number of inflorescences than the experimental groups $(1988,1993,1995,1999)$ but there was no consistent pattern of inflorescence production between the control and experimental groups, or even within the experimental treatments themselves. All groups had similar low production in some years and similar high production in others, regardless of treatment regime (Figure 1).

These results, and those reported here, will undoubtedly evoke debate within the biodynamic community over how the ash was prepared, and the susceptibility of Steiner's methods to experimental verification, though Spieß' extensive work using a variety of preparation methods on established biodynamic fields should mitigate these criticisms. Some critics will undoubtedly claim that Steiner's methods are effective, but cannot be verified in controlled experiments. These claims are in direct contradiction to Steiner's own expectations that his methods should, and would be verified experimentally [5].

In terms of ash preparation, Thun [16] has suggested that effective preparation should take both lunar position and zodiacal sign into consideration. She goes so far as to suggest that the moon's position in the zodiac is specific for a given species of weed, whose seed should only be burned when the moon is in that constellation. She also recommends that the ash be prepared in specific homeopathic potencies for effective use. Steiner's original presentation

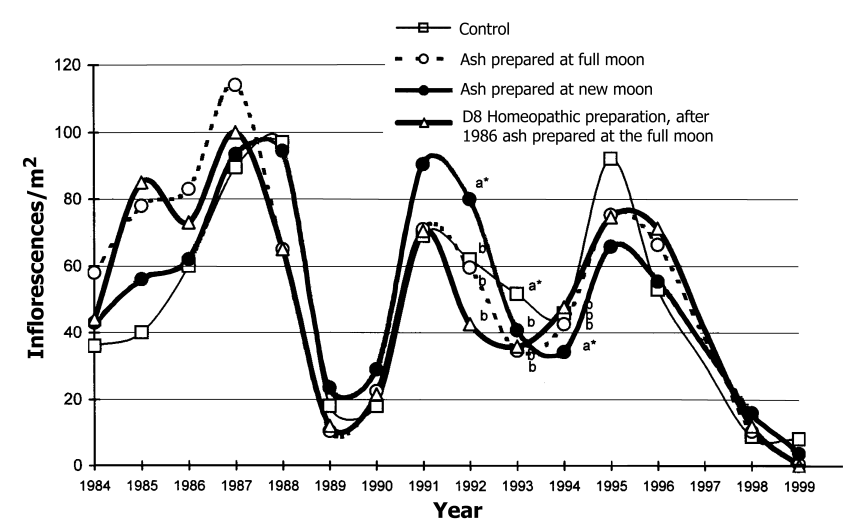

Figure 1. Results of a 17 year study (data was not collected for the first two years) on Taraxacum officinale occurrence after application of a BD seed pepper. Asterisks $\left({ }^{*}\right)$ indicate treatment/years where there was a significant difference in yield at the 0.5 level (Modified after [15], with permission). 
of his method gives no specific recommendation on either the position of the moon in the zodiac, or in fact of any astrologically beneficial configuration for burning the seeds [5]. His published method is clearly and simply stated. The farmer is to gather the seeds, burn them and spread the ash "taking no special care to do so". In handwritten notes for the lecture in which he proposed this method, Steiner did give an indication of a favorable lunar position, burning during a waning moon. These notes were published in English in the 1993 edition of his Agriculture Course, which was used as the source for our methods [5]. Recommendations on zodiacal positions and homeopathic potencies arise from Thun's own research, which has not been peer reviewed

\section{References and Notes}

[1] Westbrooks RG. Invasive plants: Changing the landscape of America: Fact Book. Washington, DC, USA: Federal Interagency Committee for the Management of Noxious and Exotic Weeds (FICMNEW); 1998.

[2] Pimentel D. Biological control of invading species. Science. 2000;289(5481):869.

[3] Tu M, Hurd C, Randall JM. Weed control methods handbook: Tools and techniques for use in natural areas. Davis, CA, USA: The Nature Conservency; 2001. Available from: http://tncweeds.ucdavis.edu/handbook.html.

[4] Pimentel D, Zuniga R, Morrison D. Update on the environmental and economic costs associated with alien-invasive species in the United States. Ecological Economics. 2005;52(3):273-288.

[5] Steiner R. Spiritual Foundations for the Renewal of Agriculture. Kimberton, PA, USA: Bio-Dynamic Farming and Gardening Association; 1924/1993.

[6] Thun M. Work on the land and the constellations. East Ginstead, England: Lanthorn Press; 1997.

[7] Williams PH, Hill CB. Rapid-cycling populations of Brassica. Science. 1986;232(4756):1385-1389.

[8] The Biology of Brassica rapa L. Canadian Food Inspection Agency; 2014. BIO1992-1902. Available from: http://www.inspection.gc.ca/plants/plantswith-novel-traits/applicants/directive-94-08/biologydocuments/brassica-rapa-l-/eng/1330965093062/ $1330987674945 \#$ b3.

[9] IBM SPSS Statistics for Windows version 19.0. Ar-
[16]. We find Thun's recommendations implausible, given that Steiner's much simpler method has failed to yield positive results, and given Spieß' failure to achieve results using homeopathically prepared peppers (Figure 1) [15].

\section{Acknowledgements}

I thank Melanie Eldridge for conducting these experiments and writing an early draft of this manuscript, in partial fulfillment of the requirements for an independent research course at the University of North Carolina at Greensboro. I regret that Melanie could not be contacted to grant consent to be a co-author of this paper.

monk, NY, USA; 2010.

[10] IBM SPSS Statistics for Windows version 22.0. Armonk, NY, USA; 2013.

[11] Eldridge M, Kirchoff BK, Richter SJ. An experimental test of the biodynamic plant peppers. Biodynamics. 2005;254:30-32.

[12] Eldridge M, Kirchoff BK, Richter SJ. A further test of the biodynamic plant peppers. Biodynamics. 2006;255:37-40.

[13] Eason CT, Hickling GJ. Evaluation of a Biodynamic Technique for Possum Pest-Control. New Zealand Journal of Ecology. 1992;16(2):141-144.

[14] Bächi-Kunz R. Untersuchungen über Anwendungen der Veraschung nach R. Steiner (1924) im Zusammenhang mit kosmischen Konstellationen am Beispiel von Tribolium castaneum Herbst (Col: Tenebrionidae) [PhD Thesis]. Eidgenössische Technische Hochschule, Zürich, Switzerland; 1985.

[15] Spieß H. In: Zur praktischen Anwendung kosmischer Rhythmen im biologisch-dynamischen Pflanzenbau. Untersuchungen zur Unkrautregulierung mit der Veraschungsmethode nach Rudolf Steiner. Demeter Hessen e.V.;. p. 1-12. Available from: http://www.dottenfelderhof.de/fileadmin/ images/forschung/Veroeffentlichungen/Zuechtung/ Veraschungsmethode_1999.pdf.

[16] Thun M, Heinze H. Mondrhythmen im Siderischen Umlauf und Pflanzenwachstum. Darmstadt, Germany: Forschungsring für Biologisch-Dynamische Wirtschaftsweise; 1979. 


\section{A Review of 'Organic Struggle: The Movement for Sustainable Agriculture in the United States'}

Published: 31 May 2016

Keywords: big organic; local food systems; organic certification; organic farming; organic food economics

Organic Struggle: The Movement for Sustainable Agriculture in the United States

Obach BK

MIT Press: Cambridge, MA, USA. 2015

327 pp.; ISBN: 978-0-262-02909-4

Organic Struggle chronicles the challenges encountered by innovators in a growing segment of the U.S. food production and marketing system. Practiced for millenia by farmers before the introduction of chemical fertilizers and pesticides, and first developed more formally in Europe, organic farming practices began to gain prominence in the U.S. only in the 1950s. Far more than a system for producing food, this strategy has become a focus for those supporting healthy and pesticide-free products, for some who embrace the organic system as a food movement, and by many who disagree with the current domination of the country's food industry by large farms and a small number of multinational corporations. Within the organic sector there is debate between those who favor a system primarily run by local farmers who sell through small markets and CSAs, and others who insist that the 'Big-Organic' segment that now sells more than half of all organic food is doing more to help the environment in the large picture. Author Brian Obach describes this ongoing struggle.

Modern origins of formalized organic agriculture are thoughtfully traced to systems developed in northern Europe, especially in German-speaking Austria, Switzerland and Germany in the first half of the last century. Among the foundations for this movement are key research publications from Albert Howard based on experiences in India, the widely-cited lectures of Rudolph Steiner on biodynamic farming methods, and the reports of the Soil Association in
England. Notably missing from the chapter on early work in Europe is the excellent recent review Organic Farming: an International History by William Lockeretz [1]. Connections with religious beliefs and spirituality were seen as detrimental to acceptance of organic farming methods by some in the research establishment.

Popularizing organic farming in the U.S. is credited to J.I. Rodale and his son Robert Rodale, with the publication Organic Farming and Gardening [2] by Rodale Press and pragmatic research and demonstrations at the research center in Pennsylvania. Publication of Silent Spring by Rachel Carson [3] provided further impetus to this movement, one that became associated with innovative restaurants, farmers markets, and a diverse array of direct purchasing arrangements between farmers and consumers. A part of the 'struggle' is still associated with social justice, concerns about poverty, and preserving small farms.

Growing concern about the veracity of advertising of organic products in the marketplace led to calls for government certification of the production process. As described in Chapter 3, early statewide organizations in Maine and California in the early 1970s set the stage for wider conversations, and formation of the International Federation of Organic Agriculture Movements (IFOAM) in 1972 provided a global platform for discussion and an attempt at uniform standards. There were some cases of reported fraud in the system, along with several food scares in the 1980s that promoted action by the government. After protracted negotiation, a national organic standard was authorized in 1990 by the U.S. Congress, but it would be more than a decade before agreement was reached on the National Organic Program (NOP) that was enacted into law in 2002.

Moving back to the process, a continuing struggle between 'Big Organic' and various coalitions of small farm groups is described as the various factions debated the standards to be adopted, materials to be accepted in or- 
ganic farming, and the importance of independent oversight of the certification process. The two sides appear to have coalesced around the Organic Trade Association (OTA), representing big business, and the Organic Consumers Association (OCA) and Cornucopia Institute representing small farmers and concerned consumers. This oversimplifies the organizational landscape of organic groups, and Chapter 4 provides a more in-depth look at the genesis of several groups and the ongoing debates. The next chapter provides additional discussion of the criticisms of organic certification rules as well as concerns about health, continuing threats to the integrity of the original program, and the ever-present pressure of large industry to relax the standards to help them gain access to the continually growing market demand for organic food.

Treatment of labor, food justice, and distribution of benefits in the organic food system are explored in Chapter 6 . There is widespread concern that this niche food product has become available only to the elite, with prices often above those of conventional food in the marketplace. There is an emergence of competing groups, such as Certified Natural Growers (CNG) that uses a network of farmers who certify each other to avoid the federal bureaucracy and rigidity of the current NOP and independent certification system. A number of strategic initiatives from farmers and smaller states have expanded the options for farmers as well as consumers, and the overall effort is to create credibility in the way that food is produced and advertised.

A concluding chapter convinces the reader that the dynamic process of organic certification is still a work in

\section{References}

[1] Lockeretz W. Organic Farming: An International History. Oxfordshire, UK: CAB International; 2007. progress, with competing certifiers and divergent philosophies. An aspect not addressed in enough detail is the importance of organic exports from this country and especially imports from other places where certification may be in place but at times suspect in terms of the rigor of reviewing the production process and use of questionable inputs. Most certification organizations, public or private in the U.S. provide opportunity for farmers to certify for markets outside the U.S., particularly those in E.U., Japan, and Canada. This is a dynamic industry where there is remarkable room for innovation, and a segment of the food industry that continues to grow in response to consumer demand.

In conclusion, Organic Struggle is a book well worth reading to be able to grasp an overview of a complicated part of our contemporary food system. With ongoing debates, competing organizations, and a skeptical public that tries to understand this complex food landscape when making purchasing decisions, the book provides factual information and many quotes from farmers and other participants that delivers a rich resource to help the reader understand the organic farming and food system.

\section{Charles Francis}

Department of Agronomy \& Horticulture, University of Nebraska - Lincoln, Lincoln, NE, USA; and Plant Sciences Department, Norwegian University of Life Sciences, Ås, Norway; E-Mail: charf@nmbu.no

[2] Rodale JI, Rodale R. Organic Farming and Gardening. Emmaus, PA, USA: Rodale Press; 1942.

[3] Carson R. Silent Spring. New York, NY, USA: Ballantine Books, Random House Publishers; 2002. 


\title{
Can the Adoption of Organic Farming Be Predicted by Biogeographic Factors? A French Case Study
}

\author{
Marco Pautasso ${ }^{1, \dagger, *}$, Anja Vieweger ${ }^{2}$ and A. Márcia Barbosa ${ }^{3}$ \\ ${ }^{1}$ Animal and Plant Health Unit, European Food Safety Authority (EFSA), Parma, Italy \\ 2 Organic Research Centre, Elm Farm, Hamstead Marshall, Newbury, UK \\ ${ }^{3}$ Centro de Investigação em Biodiversidade e Recursos Genéticos (CIBIO), InBIO Research Network in Biodiversity and \\ Evolutionary Biology, University of Évora, Portugal \\ * Corresponding author: E-Mail: marpauta@gmail.com; Tel.: +39 521036775 \\ $\dagger$ The positions and opinions presented in this article are those of the authors alone and are not intended to represent the \\ views or scientific works of EFSA.
}

Submitted: 13 January 2016 | In revised form: 21 April 2016 | Accepted: 9 June 2016 | Published: 29 June 2016

\begin{abstract}
Organic farming adoption is on the rise in many countries, due to the increased awareness of farmers, citizens, governments and other stakeholders of its more sustainable nature. Various studies have investigated the socio-economic drivers (e.g., consumer demand, support measures, agricultural policies) of organic farming adoption, but less attention has been paid to whether biogeographic factors could also be associated with variation in rates of organically managed farms in certain regions within countries. We investigate whether biogeographic factors are associated with variation in the proportion of land under organic farming in French departments. The proportion of land under organic farming increased with decreasing latitude and increasing department area. Non-significant factors were number of plant taxa, proportion of Natura 2000 protected areas, connectivity, longitude, altitude and department population. These results were robust to controlling for spatial autocorrelation. Larger and southern French departments tend to have a greater adoption of organic farming, possibly because of the more extensive nature of agriculture in such regions. Biogeographic factors have been relatively neglected in investigations of the drivers of organic farming adoption, but may have an important explanatory value.
\end{abstract}

Keywords: biodiversity; France, human population; land sharing; macroecology; organic farming; plant species richness; protected areas; spatial autocorrelation; sustainable development

\section{Introduction}

Organic farming is on the rise globally [1]. Between 2001 and 2011, agricultural land under organic management increased from nearly 16 to over 37 million hectares worldwide [2]. This trend is also reflected in the market for organ- ically grown produce; during the same decade, the global organic market grew by $170 \%$, with sales reaching nearly 63 billion US\$ in 2011 [3]. In 2011, France (3.8 billion Euros) was the second largest market for organic products in Europe (21.5 billion Euros) [4].

Organic farming aims to reconnect agriculture with na- 
ture and biodiversity, making use of natural systems and cycles and reducing external inputs (for example, growing a wider variety of crops and using natural ways to reduce pest populations, e.g. rotations). Industrial agriculture is currently one of the greatest threats to biodiversity [5-7]. Several studies have shown that organic farming benefits biodiversity, because of its less intensive cultivation practices [8-14] (see also [15]).

So far, studies investigating factors driving organic farming adoption have focused on socio-economic factors (e.g., agricultural policies, consumer demand, support measures and networks) [16-19]. However, given the connection of organic farming with nature, it could also be expected that the presence of organic farming co-varies with regional variation in biodiversity, as was shown at the landscape level [20]. Given that large-scale variation in biodiversity is in turn associated with biogeographic factors such as latitude, area and human population [21-24], it is reasonable to expect that also regional patterns in organic farming adoption will tend to be associated with such biogeographic factors.

This study aims to test this hypothesis by using data on organic farming adoption from French departments, together with some key biogeographic factors. Our main question was: is organic farming more likely to be adopted in regions with an already higher presence of biodiversity? More generally, can biogeographic factors help predict patterns in organic farming adoption across regions?

\section{Material and Methods}

Data on the proportion of agricultural land under organic farming (2008) for the 96 French metropolitan departments (with exception of Paris: data not available) were obtained from the website for sustainable development of the French government (http://www.statistiques. developpement-durable.gouv.fr/). From the same website, data for each French department were obtained on landscape connectivity (average size of natural habitats; 2006), the proportion of Natura 2000 protected areas (2009), the total area and human population (2011). Altitude was obtained from [25] as a raster map at ca. $1 \mathrm{~km}^{2}$ resolution, and averaged at French departments using the zonal statistics plugin of QGIS 2.6 [26] and an administrative map downloaded from the EDIT Geoplatform [27]. Natura 2000 data are indicators of recent efforts to achieve nature protection and may not be representative of historic or overall actions to protect nature, as the Natura 2000 reserve selection focused on regions with low presence of already available protection (i.e. National and Regional parks). Data on the number of vascular plant taxa (including subspecies) recorded for each department were obtained in 2012 from the Tela Botanica website (http://www.telabotanica.org/page:chorologie?format=html). Given the relatively low number of data points, we avoided including an excessive number of explanatory variables; further biogeographic factors could be considered in future analyses, including distance from the sea and road density.

Multivariate models were run in Spatial Analysis for Macroecology (SAM) [28]. Given that spatial autocorrelation can reduce the effective degrees of freedom, thus leading to potentially misleading $P$-values [29], the analysis was performed both without (Linear Regression Model) and with controlling (Spatial Autoregression, Generalized Least Squares, with a Gaussian Model for the residual spatial component) for spatial autocorrelation $[30,31]$. All variables (apart from latitude and longitude) were log-transformed prior to analysis so as to better approach a normal distribution. Non-significant variables (at $p>0.05$ ) were kept in the models to demonstrate that they were not significant predictors. The significance of the significant factors was robust against model reduction. We did not observe strong collinearity (correlation coefficient $>0.70$ ) among the explanatory variables, with the only exception of connectivity and plant biodiversity (correlation coefficient $=0.75$ ).

\section{Results}

Without controlling for spatial autocorrelation, the proportion of organic farming in French departments increased significantly with decreasing latitude and increasing department area. There was no significant association with plant biodiversity, proportion of protected areas, connectivity, longitude, altitude and human population size (Table 1).

All these results were confirmed when controlling for spatial autocorrelation, although with slightly different $P$-values and parameter estimates (Table 2). On its own, latitude explains about $40 \%$ of the variation among French departments in their proportion of organic farming (Figure 1). Department area on its own explains about $17 \%$ of the variation in proportion of organic farming, but this is largely due to a few data points, i.e. some small departments in the lle-de-France area with very low proportion of organic farming.

Table 1. Results of a General Linear Model for the proportion of agricultural land under organic farming in French Departments (2008) as a function of plant biodiversity, landscape connectivity, proportion of Natura 2000 protected areas, latitude, longitude, altitude, human population size and department area. The number of data points is 95 , the adjusted $\mathrm{R}^{2}$ of the model 0.50 , and the intercept 1.457 (s.e. $=2.074$ ).

\begin{tabular}{lrrrrrrrr}
\hline & N of plant taxa & Landscape connectivity & $\%$ Natura 2000 & Latitude & Longitude & Altitude & Human population & Area \\
\hline parameter estimate & 0.264 & 0.047 & 0.113 & -0.084 & 0.003 & 0.038 & 0.056 & 0.327 \\
s.e. & 0.486 & 0.101 & 0.09 & 0.024 & 0.017 & 0.172 & 0.132 & 0.139 \\
$P$-value & 0.59 & 0.64 & 0.21 & $<\mathbf{0 . 0 0 1}$ & 0.87 & 0.82 & 0.67 & $\mathbf{0 . 0 2}$ \\
\hline
\end{tabular}


Table 2. Results of a Generalized Least Squares model controlling for spatial autocorrelation, for the proportion of agricultural land under organic farming in French Departments (2008) as a function of plant biodiversity, landscape connectivity, proportion of Natura 2000 protected areas, latitude, longitude, altitude, human population size and department area. The number of data points is 95 , the Akaike Criterion Indicator of the model 59.9, and the intercept 1.457 (s.e. $=1.972$ ).

\begin{tabular}{lrrrrrrrr}
\hline & N of plant taxa & Landscape connectivity & $\%$ Natura 2000 & Latitude & Longitude & Altitude & Human population & Area $\left(\mathrm{km}^{2}\right)$ \\
\hline Parameter estimate & 0.264 & 0.047 & 0.113 & -0.084 & 0.003 & 0.038 & 0.056 & 0.327 \\
s.e. & 0.462 & 0.096 & 0.086 & 0.023 & 0.016 & 0.164 & 0.125 & 0.133 \\
$P$-value & 0.57 & 0.63 & 0.19 & $<\mathbf{0 . 0 0 1}$ & 0.87 & 0.81 & 0.65 & $\mathbf{0 . 0 2}$ \\
\hline
\end{tabular}

\section{Discussion}

Several studies have shown the essential role of agroecological approaches, and particularly organic agriculture, for sustainable development [32-34]. Ecological intensification enables an improvement of productivity while at the same time reducing adverse effects on the environment [35-38]. Not only the adoption of organic farming practices, but also research on organic farming has expanded considerably over the last years [20,39-41]. This study provides evidence that biogeographic factors can be associated with patterns in organic farming adoption across regions.

There are three main results of this analysis. First, there are no substantial differences between models of the proportion of organic farming in French departments (i) taking spatial autocorrelation into account, and (ii) not taking it into account. The results of models taking into account spatial correlation are more conservative and should be trusted more than those without taking it into account, but in this case there are only slight differences in parameter estimates and $P$-values.

Second, this result does not imply that there is no spatial autocorrelation in the examined variables. For example, the investigated response variable (the proportion of cultivated land under organic farming) was significantly spatially autocorrelated at short distances, as shown by an analysis of Moran's I (Figure 2). This result is in agreement with previous reports of spatial aggregation and neighbouring effects in the adoption of organic farming within countries [42-45]. To some extent, such spatial aggregation might be due to the underlying spatial autocorrelation of biogeographic factors associated with variation in organic farming adoption. However, there is also an important role of neighbouring effects of e.g. social networks and farmer communities in explaining the spatial aggregation of organic farming.

A third result of this study (which holds when controlling for spatial autocorrelation) is the latitudinal gradient from North to South in French adoption of organic farming. Farmers in the South of France might have switched more easily to organic cultivation because of the larger variety of crops they can cultivate in their climatic and environmental settings. In addition, it could be easier to switch to organic cultivation in viticulture (which is typical in Southern France) than for other crops. Moreover, due to a mix of climatic, edaphic, historical and cultural reasons, Southern French departments tend to be located in regions of less intensive agriculture, thus facilitating the adoption of less intensive agricultural practices [46]. This finding is in agreement with previous analyses in England, Germany, the USA and Sweden, which found that organic farming was more likely to occur in marginal areas, where the loss of production due to organic conversion is relatively small $[20,47,48]$ and in regions with more heterogeneous landscapes [49], thus likely to harbour greater plant biodiversity [50]. However, we did not observe a significant association of organic farming adoption with plant biodiversity. It is also possible that the observed latitudinal gradient in organic farming adoption correlates with other socio-economic factors (e.g. personal beliefs, levels of political and financial support, public perceptions, type of crops) that were not considered here.

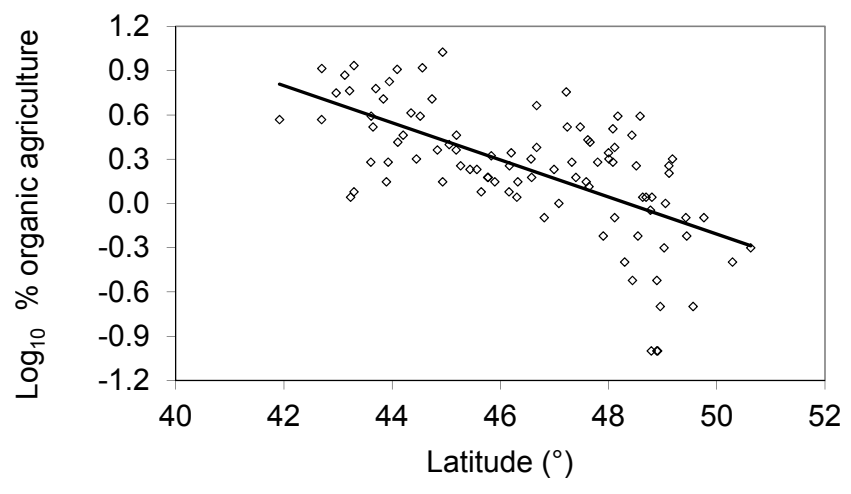

Figure 1. Latitudinal gradient of the proportion of agricultural land under organic farming (logarithmically transformed) in French departments (2008; $\mathrm{n}=95, y=$ $\left.-0.125 x+6.079, R^{2}=0.40, p<0.001\right)$.

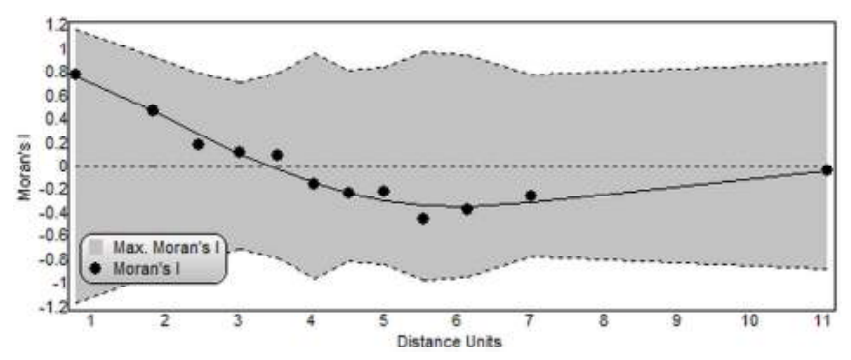

Figure 2. Moran's I for the proportion of agricultural land under organic farming (logarithmically transformed) in French departments (2008). 
Interestingly, although there is generally little variation among French departments in area, this was a significant factor in our analysis, due to the few very small departments (mainly located in the Paris area) having surprisingly low organic farming adoption rates.

Further research should investigate whether biogeographic factors are still significant determinants of organic farming adoption when including a large suite of socioeconomic explanatory variables. Econometric and socioeconomic models of organic farming adoption may benefit from including data on large-scale biogeographic factors. It would also be interesting to use a finer spatial resolution of the data used here, to test for any scale-dependence in the relative importance of biogeographic and socio-economic factors as explanatory variables for organic farming adoption rates.

\section{Conclusions}

Most research on regional patterns of organic farming has focused on socio-economic and cultural factors, from policy support to agglomeration effects and from the philosophy of farmers to the development of markets for organic produce and organic seed [40,51-59]. Whilst these factors are undoubtedly important, this study builds on evidence obtained at the landscape level on the role of environmental factors in shaping organic farming adoption $[20,49]$ and suggests that biogeographic variables may play a contributing role in how widespread organic farming is becoming across entire countries.

\section{Acknowledgements}

Many thanks to V. Chable, O. Holdenrieder, D. McKey and S. Vos for insights and discussions, and to T. Döring, T. Matoni and anonymous reviewers for helpful comments on a previous draft. AMB is supported by Fundação para a Ciência e a Tecnologia through 'FCT Investigator' contract IF/00266/2013 and exploratory project CP1168/CT0001.

\section{References and Notes}

[1] Granatstein D, Kirby E, Willer H, et al. Organic horticulture expands globally. Chronica Horticulturae. 2010;50(4):31-38.

[2] Paull J. The uptake of organic agriculture: A decade of worldwide development. Journal of Social and Development Sciences. $2011 ; 2(3): 111-120$.

[3] Willer H, Lernoud J, Home R. The world of organic agriculture 2013: Summary. In: Willer H, Lernoud J, Kilcher L, editors. The World of Organic Agriculture. Statistics and Emerging Trends. Frick, Switzerland: Research Institute of Organic Agriculture (FiBL) and International Federation of Organic Agriculture Movements (IFOAM); 2013. pp. 26-33.

[4] Schaack D PSWH Lernoud J. The organic market in Europe 2011Nine percent increase compared with 2010. In: Willer H, Lernoud J, Kilcher L, editors. The World of Organic Agriculture. Statistics and Emerging Trends. Frick, Switzerland: Research Institute of Organic Agriculture (FiBL) and International Federation of Organic Agriculture Movements (IFOAM); 2013. pp. 224-229.

[5] Donald PF, Green RE, Heath MF. Agricultural intensification and the collapse of Europe's farmland bird populations. Proceedings of the Royal Society of London B: Biological Sciences. 2001;268(1462):2529. doi:10.1098/rspb.2000.1325.

[6] Stoate C, Báldi A, Beja P, Boatman ND, Herzon I, van Doorn A, et al. Ecological impacts of early 21 st century agricultural change in Europe - A review. Journal of Environmental Management. 2009;91(1):22-46. doi:10.1016/j.jenvman.2009.07.005.

[7] Balmford A, Green R, Phalan B. What conservationists need to know about farming. Proceedings of the Royal Society B: Biological Sciences. 2012;279(1739):2714-2724. doi:10.1098/rspb.2012.0515.

[8] Mader P. Soil fertility and biodiversity in organic farming. Science. 2002;296(5573):1694-1697. doi:10.1126/science.1071148.

[9] Gabriel D, Tscharntke T. Insect pollinated plants benefit from organic farming. Agriculture, Ecosystems \& Environment. 2007;118(1-4):4348. doi:10.1016/j.agee.2006.04.005.

[10] Holzschuh A, Steffan-Dewenter I, Tscharntke T. Agricultural landscapes with organic crops support higher pollinator diversity. Oikos. 2008;117(3):354-361. doi:10.1111/j.2007.0030-1299.16303.x.

[11] Chifflot V, Rivest D, Olivier A, Cogliastro A, Khasa D. Molecular analysis of arbuscular mycorrhizal community structure and spores distribution in tree-based intercropping and forest systems. Agriculture, Ecosystems \& Environment. 2009;131(1-2):32-39. doi:10.1016/j.agee.2008.11.010.

[12] Rundlöf M, Edlund M, Smith HG. Organic farming at local and land- scape scales benefits plant diversity. Ecography. 2009;33(3):514-522. doi:10.1111/j.1600-0587.2009.05938.x.

[13] Tuomisto HL, Hodge ID, Riordan P, Macdonald DW. Does organic farming reduce environmental impacts? - A meta-analysis of European research. Journal of Environmental Management. 2012;112:309-320. doi:10.1016/j.jenvman.2012.08.018.

[14] Tuck SL, Winqvist C, Mota F, Ahnström J, Turnbull LA, Bengtsson J. Land-use intensity and the effects of organic farming on biodiversity: a hierarchical meta-analysis. Journal of Applied Ecology. 2014;51(3):746-755. doi:10.1111/1365-2664.12219.

[15] Schneider MK, Lüscher G, Jeanneret P, Arndorfer M, Ammari Y, Bailey $\mathrm{D}$, et al. Gains to species diversity in organically farmed fields are not propagated at the farm level. Nature Communications. 2014;5. doi:10.1038/ncomms5151.

[16] Lamine C, Bellon S. Conversion to organic farming: a multidimensional research object at the crossroads of agricultural and social sciences. A review. Agronomy for Sustainable Development. 2009;29(1):97-112. doi:10.1051/agro:2008007.

[17] Schmidtner E, Lippert C, Dabbert S. Haben Nachbarschaftseffekte einen Einfluss auf die räumliche Verteilung des Öko-Landbaus in Deutschland? 20. Jahrestagung der Österreichischen Gesellschaft für Agrarökonomie. In: Land-und Ernährungswirtschaft 2020. Vienna, Austria: Universität für Bodenkultur; 2010. pp. 109-110.

[18] Kaufmann P, Zemeckis R, Skulskis V, Kairyte E, Stagl S. The diffusion of organic farming in Lithuania. Journal of Sustainable Agriculture. 2011;35(5):522-549. doi:10.1080/10440046.2011.579838.

[19] Ilbery B, Kirwan J, Maye D. Explaining regional and local differences in organic farming in England and Wales: A comparison of South West Wales and South East England. Regional Studies. 2014;50(1):110-123. doi:10.1080/00343404.2014.895805.

[20] Gabriel D, Carver SJ, Durham H, Kunin WE, Palmer RC, Sait SM, et al. The spatial aggregation of organic farming in England and its underlying environmental correlates. Journal of Applied Ecology. 2009;46(2):323-333. doi:10.1111/j.1365-2664.2009.01624.x.

[21] Bernholt H, Kehlenbeck K, Gebauer J, Buerkert A. Plant species richness and diversity in urban and peri-urban gardens of Niamey, Niger Agroforestry Systems. 2009;77(3):159-179. doi:10.1007/s10457009-9236-8.

[22] Pecher C, Fritz SA, Marini L, Fontaneto D, Pautasso M. Scaledependence of the correlation between human population and the species richness of stream macro-invertebrates. Basic and Applied Ecology. 2010;11(3):272-280. doi:10.1016/j.baae.2009.09.005.

[23] Freeman J. Domesticated crop richness in human subsistence cul- 
tivation systems: a test of macroecological and economic determinants. Global Ecology and Biogeography. 2011;21(4):428-440. doi:10.1111/j.1466-8238.2011.00687.x.

[24] de Grenade R, Nabhan GP. Baja California peninsula oases: An agro-biodiversity of isolation and integration. Applied Geography. 2013;41:24-35. doi:10.1016/j.apgeog.2013.03.008

[25] Hijmans RJ, Cameron SE, Parra JL, Jones PG, Jarvis A. Very high resolution interpolated climate surfaces for global land areas. International Journal of Climatology. 2005;25(15):1965-1978. Data available from: http://www.worldclim.org. doi:10.1002/joc.1276.

[26] QGIS Development Team. QGIS Geographic Information System; 2014. Available from: http://qgis.osgeo.org.

[27] Sastre P, Roca P, Lobo JM. A Geoplatform for improving accessibility to environmental cartography. Journal of Biogeography. 2009;36(3):568-568. doi:10.1111/j.1365-2699.2008.02070.x

[28] Rangel TF, Diniz-Filho JAF, Bini LM. SAM: a comprehensive application for Spatial Analysis in Macroecology. Ecography. 2010;33(1):4650. doi:10.1111/j.1600-0587.2009.06299.x

[29] Dormann CF. Effects of incorporating spatial autocorrelation into the analysis of species distribution data. Global Ecology and Biogeography. 2007;16(2):129-138. doi:10.1111/j.1466-8238.2006.00279.x.

[30] Pautasso M, Zotti M. Macrofungal taxa and human population in Italy's regions. Biodiversity and Conservation. 2008;18(2):473-485. doi:10.1007/s10531-008-9511-4.

[31] Cantarello E, Steck CE, Fontana P, Fontaneto D, Marini L, Pautasso M. A multi-scale study of Orthoptera species richness and human population size controlling for sampling effort. Naturwissenschaften. 2009;97(3):265-271. doi:10.1007/s00114-009-0636-4.

[32] Geertsema W, Rossing WA, Landis DA, Bianchi FJ, van Rijn PC, Schaminée $\mathrm{JH}$, et al. Actionable knowledge for ecological intensification of agriculture. Frontiers in Ecology and the Environment. 2016;14(4):209-216. doi:10.1002/fee.1258.

[33] Gomiero T, Pimentel D, Paoletti MG. Environmental impact of different agricultural management practices: Conventional vs. organic agriculture. Critical Reviews in Plant Sciences. 2011;30(1-2):95-124. doi:10.1080/07352689.2011.554355.

[34] Wolf BM, Häring AM, Heß J. Strategies towards evaluation beyond scientific impact. Pathways not only for agricultural Research. Organic Farming. 2015;1(1). doi:10.12924/of2015.01010003.

[35] Watson CA, Atkinson D, Gosling P, Jackson LR, Rayns FW. Managing soil fertility in organic farming systems. Soil Use and Management. 2006;18:239-247. doi:10.1111/j.1475-2743.2002.tb00265.x.

[36] Kassam A, Friedrich T. Nutrient management in conservation agriculture: a biologically-based approach to sustainable production intensification. In: 7th Conservation Agriculture Conference. Dnipropetrovsk, Ukraine; 2009. pp. 1-20.

[37] Scialabba NEH, Müller-Lindenlauf M. Organic agriculture and climate change. Renewable Agriculture and Food Systems. 2010;25(02):158169. doi:10.1017/s1742170510000116.

[38] Smith LG, Williams AG, Pearce BD. The energy efficiency of organic agriculture: A review. Renewable Agriculture and Food Systems. 2014;30(03):280-301. doi:10.1017/s1742170513000471.

[39] Siegmeier T, Möller D. Mapping research at the intersection of organic farming and bioenergy - A scientometric review. Renewable and Sustainable Energy Reviews. 2013;25:197-204. doi:10.1016/j.rser.2013.04.025.

[40] Döring TF. A fresh start for organic farming research. 2013;1(1):1-2. doi:10.12924/of2014.01010001.

[41] Aleixandre JL, Aleixandre-Tudó JL, Bolaños-Pizarro M, AleixandreBenavent R. Mapping the scientific research in organic farming: a bibliometric review. Scientometrics. 2015;105(1):295-309. doi:10.1007/s11192-015-1677-4.

[42] Schmidtner E, Lippert C, Engler B, Haring AM, Aurbacher J, Dabbert S. Spatial distribution of organic farming in Germany: does neighbourhood matter? European Review of Agricultural Economics.
2011;39(4):661-683. doi:10.1093/erae/jbr047.

[43] Lapple D, Kelley H. Spatial dependence in the adoption of organic drystock farming in Ireland. European Review of Agricultural Economics. 2014;42(2):315-337. doi:10.1093/erae/jbu024.

[44] Wollni M, Andersson C. Spatial patterns of organic agriculture adoption: Evidence from Honduras. Ecological Economics. 2014;97:120128. doi:10.1016/j.ecolecon.2013.11.010.

[45] Yang AL, Rounsevell MDA, Wilson RM, Haggett C. Spatial analysis of agri-environmental policy uptake and expenditure in Scotland. Journal of Environmental Management. 2014;133:104-115. doi:10.1016/j.jenvman.2013.11.038.

[46] Teillard F, Allaire G, Cahuzac E, Léger F, Maigné E, Tichit M. A novel method for mapping agricultural intensity reveals its spatial aggregation: Implications for conservation policies. Agriculture, Ecosystems \& Environment. 2012;149:135-143. doi:10.1016/j.agee.2011.12.018.

[47] Kostandini G, Mykerezi E, Tanellari E. Viability of organic production in rural counties: county and state-level evidence from the United States. Journal of Agricultural and Applied Economics. 2011;43(03):443-451.

[48] Rundölf M, Smith HG. The effect of organic farming on butterfly diversity depends on landscape context. Journal of Applied Ecology. 2006;43(6):1121-1127. doi:10.1111/j.1365-2664.2006.01233.x.

[49] Norton L, Johnson P, Joys A, Stuart R, Chamberlain D, Feber R, et al. Consequences of organic and non-organic farming practices for field, farm and landscape complexity. Agriculture, Ecosystems \& Environment. 2009;129(1-3):221-227. doi:10.1016/j.agee.2008.09.002.

[50] Bredemeier B, Rüter S, von Haaren C, Reich M, Schaarschmidt F Spatial congruence between organic farming and biodiversity related landscape features in Germany. International Journal of Biodiversity Science, Ecosystem Services \& Management. 2015;11(4):330-340. doi:10.1080/21513732.2015.1094515.

[51] Padel S. Conversion to organic farming: A typical example of the diffusion of an innovation? Sociologia Ruralis. 2001;41(1):40-61. doi:10.1111/1467-9523.00169.

[52] Koesling $\mathrm{M}$, Flaten $\mathrm{O}$, Lien $\mathrm{G}$. Factors influencing the conversion to organic farming in Norway. IJARGE. 2008;7(1-2):78-95. doi:10.1504/ijarge.2008.016981.

[53] Geniaux G, Lambert M, Bellon S. Analyse de la diffusion spatiale de l'agriculture biologique en région Provence-Alpes-Côte d'Azur (Paca): construction d'une méthodologie d'observation et de prospective. Innovations Agronomiques. 2009;4:417-426.

[54] Döring TF, Bocci R, Hitchings R, Howlett S, van Bueren ETL, Pautasso $M$, et al. The organic seed regulations framework in Europe-Current status and recommendations for future development. Organic Agriculture. 2012;2(3-4):173-183. doi:10.1007/s13165-012-0034-7.

[55] Allaire G, Cahuzac É, Poméon T, Simioni M. Approche spatiale de la conversion à l'agriculture biologique. Les dynamiques régionales en France. Économie rurale. 2014;(339-340):9-31. doi:10.4000/economierurale.4200.

[56] Latruffe L, Nauges C. Technical efficiency and conversion to organic farming: the case of France. European Review of Agricultural Economics. 2013;41(2):227-253. doi:10.1093/erae/jbt024.

[57] Allaire G, Poméon T, Maigné E, Cahuzac E, Simioni M, Desjeux Y. Territorial analysis of the diffusion of organic farming in France: Between heterogeneity and spatial dependence. Ecological Indicators. 2015;59:70-81. doi:10.1016/j.ecolind.2015.03.009.

[58] Home R, Ries E, Tschanz A, Indermühle A. Social factors in the decision by Swiss farmers to convert to organic farming. Acta Fytotechnica et Zootecnica. 2015;18(Special Issue):154-156. doi:10.15414/afz.2015.18.si.154-156.

[59] Boncinelli F, Bartolini F, Brunori G, Casini L. Spatial analysis of the participation in agri-environment measures for organic farming. Renewable Agriculture and Food Systems. 2015; doi:10.1017/s1742170515000307. 
librello 4 s

\begin{abstract}
About Librello
Librello is a publishing house established in Basel, Switzerland. All content published by Librello is open access, available upon publication for any reader. We strongly believe that open access improves the exchange of scientific knowledge, and consists in a more ethic way of publishing the results of research supported by public funds.

Librello is an innovative publishing enterprise. Our novel model works on a membership basis to decouple the payment from the publication. On one side, we can afford a stringent peer-review with no economic pressure, and the authors also profit from our business model by being able to submit multiple manuscripts by a single annual fee.
\end{abstract}

Librello Publishing House

4000 Basel

Switzerland

http://librelloph.com 